\title{
INTERSECTING FAMILIES OF PERMUTATIONS
}

\author{
DAVID ELLIS, EHUD FRIEDGUT, AND HARAN PILPEL
}

\section{INTRODUCTION}

The classical Erdős-Ko-Rado theorem states that if $r<n / 2$, an intersecting family of $r$-subsets of $\{1,2, \ldots, n\}$ has size at most $\left(\begin{array}{c}n-1 \\ r-1\end{array}\right)$; if equality holds, the family must consist of all $r$-subsets containing a fixed element. The so-called 'second Erdös-Ko-Rado theorem' states that if $n$ is sufficiently large depending on $k$ and $r$, then any $k$-intersecting family of $r$-subsets of $\{1,2, \ldots, n\}$ has size at most $\left(\begin{array}{c}n-k \\ r-k\end{array}\right)$; if equality holds, the family must consist of all $r$-subsets containing $k$ fixed elements. We deal with analogues of these results for permutations.

As usual, $[n]$ will denote the set $\{1,2, \ldots, n\}$, and $S_{n}$ will denote the symmetric group, the group of all permutations of $[n]$. Two permutations $\sigma, \tau \in S_{n}$ are said to intersect if they agree at some point, i.e. if there exists $i \in[n]$ such that $\sigma(i)=\tau(i)$. Similarly, they are said to $k$-intersect if they agree on at least $k$ points, i.e., if there exist $i_{1}, i_{2}, \ldots, i_{k} \in[n]$ such that $\sigma\left(i_{t}\right)=\tau\left(i_{t}\right)$ for $t=1,2, \ldots, k$. A subset $I \subset S_{n}$ is said to be $k$-intersecting if any two permutations in $I k$-intersect. In this paper, we characterize the largest $k$-intersecting subsets of $S_{n}$ for $n$ sufficiently large depending on $k$, proving a conjecture of Deza and Frankl. We also prove a similar result concerning $k$-cross-intersecting subsets, proving a conjecture of Leader.

Our main tool in this paper is Fourier analysis on the symmetric group, which entails representation theory. Although Fourier analysis has become a central tool in combinatorics and computer science in the last two decades (notably, since the landmark paper of [20]), it has not often been applied to combinatorial problems in a non-Abelian setting. In retrospect, it seems that in this case it fits the task perfectly.

As a bonus, we point out a nice aspect of Boolean (0/1 valued) functions on $S_{n}$. One of the recurring themes in the applications of discrete Fourier analysis to combinatorics over the last decade has been showing that Boolean functions on $\{0,1\}^{n}$ are 'juntas' (i.e., depend essentially on few coordinates) precisely when their Fourier transform is concentrated mainly on small sets. Here, we study Boolean

Received by the editors March 9, 2009 and, in revised form, November 15, 2010, and December 8,2010 .

2010 Mathematics Subject Classification. Primary 05E10, 20C30, 05D99.

Key words and phrases. Intersecting families of permutations, Erdős-Ko-Rado, representation theory.

Research of the second author was supported in part by the Israel Science Foundation, grant no. 0397684, and NSERC grant 341527.

Research of the third author was supported in part by the Giora Yoel Yashinsky Memorial Grant.

(C)2011 American Mathematical Society Reverts to public domain 28 years from publication 
functions on $S_{n}$ whose Fourier transform is supported on the irreducible representations of low dimension and connect them to cosets of subgroups which are the pointwise stabilizers of small subsets of $\{1,2, \ldots, n\}$. Along the way, we also prove an interesting generalization of Birkhoff's theorem on bistochastic matrices.

\subsection{History and related results. Let}

$$
T_{i \mapsto j}=\left\{\sigma \in S_{n}, \sigma(i)=j\right\} .
$$

Clearly, $T_{i \mapsto j}$ is a 1 -intersecting subset of $S_{n}$, with size $(n-1)$ !. Let

$$
T_{i_{1} \mapsto j_{1}, \ldots, i_{k} \mapsto j_{k}}=\bigcap_{t=1}^{k} T_{i_{t} \mapsto j_{t}}=\left\{\sigma \in S_{n}: \sigma\left(i_{t}\right)=j_{t}(1 \leq t \leq k)\right\} .
$$

If $i_{1}, \ldots, i_{k}$ are distinct and $j_{1}, \ldots, j_{k}$ are distinct, then $T_{i_{1} \mapsto j_{1}, \ldots, i_{k} \mapsto j_{k}}$ is a coset of the stabilizer of $k$ points; we will refer to it as a $k$-coset.

Clearly, a $k$-coset is a $k$-intersecting family of size $(n-k)$ !. As observed by Deza and Frankl [10, it is easy to prove that a 1-intersecting subset of $S_{n}$ is no larger than a 1-coset:

Theorem 1 ([10]). For any $n \in \mathbb{N}$, if $I \subset S_{n}$ is 1-intersecting, then $|I| \leq(n-1)$ !.

Proof. Let $H$ be the cyclic group generated by the $n$-cycle $(123 \ldots n)$. No two permutations in $H$ intersect, and the same is true for any left coset of $H$. The $(n-1)$ ! left cosets of $H$ partition $S_{n}$. If $I \subset S_{n}$ is 1 -intersecting, then $I$ contains at most one permutation from each left coset of $H$, and therefore $|I| \leq(n-1)$ !.

Deza and Frankl conjectured in [10] that for any $n \in \mathbb{N}$, the 1-cosets are the only 1-intersecting subsets of $S_{n}$ with size $(n-1)$ !. Perhaps surprisingly, this turned out to be substantially harder to prove; it was first proved by Cameron and $\mathrm{Ku}$ [6] and independently by Larose and Malvenuto [21].

What about $k$-intersecting families? For $n$ small depending on $k$, the $k$-cosets need not be the largest $k$-intersecting subsets of $S_{n}$. Indeed,

$$
\left\{\sigma \in S_{n}: \sigma \text { has at least } k+1 \text { fixed points in }\{1,2, \ldots, k+2\}\right\}
$$

is a $k$-intersecting family with size

$$
(k+2)(n-k-1) !-(k+1)(n-k-2) !,
$$

which is larger than $(n-k)$ ! if $k \geq 4$ and $n \leq 2 k$. However, Deza and Frankl conjectured in [10] that if $n$ is large enough depending on $k$, the $k$-cosets are the largest $k$-intersecting subsets of $S_{n}$ :

Conjecture 1 (Deza-Frankl). For any $k \in \mathbb{N}$ and any $n$ sufficiently large depending on $k$, if $I \subset S_{n}$ is $k$-intersecting, then $|I| \leq(n-k)$ !. Equality holds if and only if $I$ is a $k$-coset of $S_{n}$.

This is our main result. Our proof uses eigenvalue techniques, together with the representation theory of $S_{n}$. In fact, it was proved by the first author and the last two authors independently in 2008. Our two proofs of the upper bound are essentially equivalent, hence the joint paper. However, the latter two authors proved the equality statement directly, via their generalization of Birkhoff's Theorem (Theorem [29), whereas the first author deduced it from the following 'stability' result, proved in 8]: 
Theorem 2 ([8]). Let $k \in \mathbb{N}$ be fixed, let $n$ be sufficiently large depending on $k$, and let $I \subset S_{n}$ be a k-intersecting family which is not contained within a $k$-coset. Then $I$ is no larger than the family

$$
\begin{gathered}
\left\{\sigma \in S_{n}: \sigma(i)=i \forall i \leq k, \sigma(j)=j \text { for some } j>k+1\right\} \\
\cup\{(1, k+1),(2, k+1), \ldots,(k, k+1)\},
\end{gathered}
$$

which has size $(1-1 / e+o(1))(n-k)$ !.

This may be seen as an analogue of the Hilton-Milner theorem [16] on intersecting families of $r$-sets; the $k=1$ case was conjectured by Cameron and $\mathrm{Ku}$ in $[\underline{6}$.

When there exists a sharply $k$-transitive subset of $S_{n}$, the 'partitioning' argument in the above proof of Theorem 1 can be replaced by a Katona-type averaging argument, proving Conjecture 1 in this case.

[Recall that a subset $T \subset S_{n}$ is said to be $k$-transitive if for any distinct $i_{1}, \ldots, i_{k} \in[n]$ and any distinct $j_{1}, \ldots, j_{k} \in[n]$, there exists $\sigma \in T$ such that $\sigma\left(i_{t}\right)=j_{t}$ for each $t \in[k] ; T$ is said to be sharply $k$-transitive if there exists a unique such $\sigma \in T$. Note that a $k$-transitive subset $T \subset S_{n}$ is sharply $k$-transitive if and only if it has size $n(n-1) \ldots(n-k+1)$.]

Indeed, suppose that $S_{n}$ has a sharply $k$-transitive subset $T$. Then any left translate $\sigma T$ of $T$ is also sharply $k$-transitive, so any two distinct permutations in $\sigma T$ agree in at most $k-1$ places. Let $I \subset S_{n}$ be $k$-intersecting; then $|I \cap \sigma T| \leq 1$ for each $\sigma \in S_{n}$. Averaging over all $\sigma \in S_{n}$ gives $|I| \leq(n-k)$ !.

For $k=2$ and $n=q$ a prime power, $S_{n}$ has a sharply 2-transitive subgroup: identify the ground set with the finite field $\mathbb{F}_{q}$ of order $q$, and take $H$ to be the group of all affine maps $x \mapsto a x+b\left(a \in \mathbb{F}_{q} \backslash\{0\}, b \in \mathbb{F}_{q}\right)$. Any two distinct permutations in $H$ agree in at most 1 point, and the same is true for any left coset of $H$. So if $I \subset S_{n}$ is 2-intersecting, then $I$ contains at most one permutation from each left coset of $H$. Since the $(n-2)$ ! left cosets of $H$ partition $S_{n}$, this implies that $|I| \leq(n-2)$ !.

Similarly, for $k=3$ and $n=q+1$ (where $q$ is a prime power), $S_{n}$ has a sharply 3 -transitive subgroup: identify the ground set with $\mathbb{F}_{q} \cup\{\infty\}$, and take $H$ to be the group of all Möbius transformations

$$
x \mapsto \frac{a x+b}{c x+d} \quad\left(a, b, c, d \in \mathbb{F}_{q}, a d-b c \neq 0\right) .
$$

However, it is a classical result of C. Jordan [19] that the only sharply $k$-transitive permutation groups for $k \geq 4$ are $S_{k}$ (for $\left.k \geq 4\right), A_{k-2}$ (for $\left.k \geq 8\right), M_{11}($ for $k=4)$ and $M_{12}$ (for $k=5$ ), where $M_{11}, M_{12}$ are the Matthieu groups. Moreover, sharply $k$-transitive subsets of $S_{n}$ have not been found for any other values of $n$ and $k$. Thus, it seems unlikely that this approach can work in general. Instead, we will use a different approach.

Recall that if $G$ is a group and $X \subset G$ is inverse-closed, the Cayley graph on $G$ generated by $X$ is the graph with vertex-set $G$ and edge-set $\left\{\{g, h\}: g h^{-1} \in\right.$ $X\}$. Let $\Gamma_{1}$ be the Cayley graph on $S_{n}$ generated by the set of fixed-point-free permutations,

$$
\mathrm{FPF}=\left\{\sigma \in S_{n}: \sigma(i) \neq i \forall i \in[n]\right\} .
$$

Note that a 1-intersecting subset of $S_{n}$ is precisely an independent set in $\Gamma_{1}$. It turns out that calculating the least eigenvalue of $\Gamma_{1}$ (meaning the least eigenvalue of its adjacency matrix) and applying Hoffman's bound (Theorem 11) yields an 
alternative proof of Theorem 1. Calculating the least eigenvalue of $\Gamma_{1}$ is nontrivial, requiring use of the representation theory of $S_{n}$. This was first done by Renteln 25], using symmetric functions, and independently and slightly later by Friedgut and Pilpel [13, and also by Godsil and Meagher [14. As observed in [13] and [14, this also leads to an alternative proof that the 1-cosets are the unique largest 1-intersecting subsets.

The obvious generalization of this approach fails for $k$-intersecting subsets of $S_{n}$. Let $\Gamma_{k}$ be the Cayley graph on $S_{n}$ generated by the set

$$
\mathrm{FPF}_{k}=\left\{\sigma \in S_{n}: \sigma \text { has less than } k \text { fixed points }\right\} \text {. }
$$

A $k$-intersecting subset of $S_{n}$ is precisely an independent set in $\Gamma_{k}$, so our task is to find the largest independent sets in $\Gamma_{k}$. Unfortunately, for $k$ fixed and $n$ large, calculating the least eigenvalue of $\Gamma_{k}$ and applying Hoffman's bound only gives an upper bound of $\Theta((n-1)$ !) on the size of a $k$-intersecting family.

A key idea of our proof is to choose various subgraphs of $\Gamma_{k}$, and to construct a 'pseudo-adjacency matrix' $A$ for $\Gamma_{k}$ which is a suitable real linear combination of the adjacency matrices of these subgraphs. We then apply a weighted version of Hoffman's Theorem (Theorem 12) to this linear combination, in order to prove the upper bound in Conjecture 1. The subgraphs chosen will be Cayley graphs generated by various unions of conjugacy classes of $S_{n}$; this will enable us to calculate their eigenvalues using the representation theory of $S_{n}$. Most of the work of the proof is in showing that an appropriate linear combination exists.

1.2. Our main results. A remark on terminology: we will often identify a subset $A$ of $S_{n}$, its characteristic function $1_{A}: S_{n} \rightarrow\{0,1\}$, and its characteristic vector $v_{A} \in \mathbb{R}^{\left|S_{n}\right|}$; so when we say that a set $A$ is spanned by sets $B_{1}, \ldots, B_{t}$, we mean that $v_{A} \in \operatorname{Span}\left\{v_{B_{1}}, \ldots, v_{B_{t}}\right\}$.

Our main results in this paper are as follows.

Theorem 3. For any $k \in \mathbb{N}$, and any $n$ sufficiently large depending on $k$, if $I \subset S_{n}$ is $k$-intersecting, then $|I| \leq(n-k)$ !. Equality holds if and only if $I$ is a $k$-coset of $S_{n}$.

We also prove a cross-intersecting version of this theorem:

Definition 1. Two sets $I, J \subset S_{n}$ are $k$-cross-intersecting if every permutation in $I k$-intersects every permutation in $J$.

Theorem 4. For any $k \in \mathbb{N}$ and any $n$ sufficiently large depending on $k$, if $I, J \subset$ $S_{n}$ are $k$-cross-intersecting, then $|I||J| \leq((n-k) !)^{2}$. Equality holds if and only if $I=J$ and $I$ is a $k$-coset of $S_{n}$.

The $k=1$ case of the above was a conjecture of Leader 22 .

Our argument proceeds in the following steps. (In order to not disrupt the flow of the paper, some of the representation-theoretic terms used will only be defined later.)

First, we bound the size of a $k$-intersecting family.

Theorem 5. For any $k \in \mathbb{N}$ and any $n$ sufficiently large depending on $k$, if $I \subset S_{n}$ is $k$-intersecting, then $|I| \leq(n-k)$ !. Moreover, if $I, J \subset S_{n}$ are $k$-cross-intersecting, then $|I||J| \leq((n-k) !)^{2}$. 
Next, we describe the Fourier transform of the characteristic functions of the families which achieve this bound. Let $V_{k}$ be the linear subspace of real-valued functions on $S_{n}$ whose Fourier transform is supported on irreducible representations corresponding to partitions $\mu$ of $n$ such that $\mu \geq\left(n-k, 1^{k}\right)$, where $\geq$ denotes the lexicographic order (see section 3).

Theorem 6. For $k$ fixed and $n$ sufficiently large depending on $k$, if $I \subset S_{n}$ is a $k$-intersecting family of size $(n-k)$ !, then $1_{I} \in V_{k}$. Furthermore if $I, J \subset S_{n}$ are $k$-cross-intersecting and $|I||J|=(n-k) !^{2}$, then $1_{I}, 1_{J} \in V_{k}$.

We then prove the following:

Theorem 7. $V_{k}$ is spanned by the characteristic functions of the $k$-cosets of $S_{n}$.

Finally, we complete the proof of our main theorem using the following combinatorial result:

Theorem 8. For any $k \in \mathbb{N}$, if $f$ is a Boolean function on $S_{n}$ which is spanned by the $k$-cosets, then $f$ is the characteristic function of a disjoint union of $k$-cosets.

Clearly, the last four theorems immediately imply our main result, Theorem 3 , and its cross-intersecting version, Theorem 4,

1.3. Structure of the paper. In section 2 we provide the background that we will use from general representation theory and graph theory. In section 3 we prove all necessary results and lemmas that pertain to representation theory of $S_{n}$. Section 4 ties together the results of the previous two sections in order to bound the size and provide a Fourier characterization of the largest $k$-intersecting families. Finally, in section 5 we show that this characterization holds only for $k$-cosets.

\section{BACKGROUND}

2.1. General representation theory. In this section, we recall the basic notions and results we need from general representation theory. For more background, the reader may consult 18 and 28 .

Let $G$ be a finite group, and let $F$ be a field. A representation of $G$ over $F$ is a pair $(\rho, V)$, where $V$ is a finite-dimensional vector space over $F$, and $\rho: G \rightarrow G L(V)$ is a group homomorphism from $G$ to the group of all invertible linear endomorphisms of $V$. The vector space $V$, together with the linear action of $G$ defined by $g v=\rho(g)(v)$, is sometimes called an FG-module. A homomorphism between two representations $(\rho, V)$ and $\left(\rho^{\prime}, V^{\prime}\right)$ is a linear map $\phi: V \rightarrow V^{\prime}$ such that $\phi(\rho(g)(v))=\rho^{\prime}(g)(\phi(v))$ for all $g \in G$ and $v \in V$. If $\phi$ is a linear isomorphism, the two representations are said to be equivalent, and we write $(\rho, V) \cong\left(\rho^{\prime}, V^{\prime}\right)$. If $\operatorname{dim}(V)=n$, we say that $\rho$ has dimension $n$. If $V=F^{n}$, then we call $\rho$ a matrix representation; choosing an $F$-basis for a general $V$, one sees that any representation is equivalent to some matrix representation.

The representation $(\rho, V)$ is said to be irreducible if it has no proper subrepresentation, i.e. if there is no proper subspace of $V$ which is $\rho(g)$-invariant for all $g \in G$.

The group algebra $F[G]$ denotes the $F$-vector space with basis $G$ and multiplication defined by extending the group multiplication linearly. In other words,

$$
F[G]=\left\{\sum_{g \in G} x_{g} g: x_{g} \in F \forall g \in G\right\}
$$


and

$$
\left(\sum_{g \in G} x_{g} g\right)\left(\sum_{h \in G} y_{h} h\right)=\sum_{g, h \in G} x_{g} y_{h}(g h) .
$$

Identifying $\sum_{g \in G} x_{g} g$ with the function $g \mapsto x_{g}$, we can view the vector space $F[G]$ as the space of all $F$-valued functions on $G$. The representation defined by

$$
\rho(g)(x)=g x \quad(g \in G, x \in F[G])
$$

is called the left regular representation of $G$; the corresponding $F G$-module is called the group module. This will be useful when we describe irreducible representations of $S_{n}$.

When $F=\mathbb{R}$ or $\mathbb{C}$, it turns out that there are only finitely many equivalence classes of irreducible representations of $G$, and any representation of $G$ is isomorphic to a direct sum of irreducible representations of $G$. Hence, we may choose a set of representatives $R$ for the equivalence classes of complex irreducible representations of $G$. For the rest of section $2, R$ will be fixed and will consist of matrix representations.

If $(\rho, V)$ is a representation of $G$, and $\alpha$ is a linear endomorphism of $V$, we say that $\alpha$ commutes with $\rho$ if $\alpha \circ(\rho(g))=\rho(g) \circ \alpha$ for every $g \in G$. (So an isomorphism of $(\rho, V)$ is simply an invertible linear endomorphism which commutes with $\rho$.) We will make use of the following:

Lemma 1 (Schur's Lemma). If $G$ is a finite group, and $(\rho, V)$ is a complex irreducible representation of $G$, then the only linear endomorphisms of $V$ which commute with $\rho$ are scalar multiples of the identity.

If $F=\mathbb{R}$ or $\mathbb{C}$, we may define an inner product on $F[G]$ as follows:

$$
\langle\phi, \psi\rangle=\frac{1}{|G|} \sum_{g \in G} \phi(g) \overline{\psi(g)} .
$$

If $(\rho, V)$ is a complex representation of $V$, the character $\chi_{\rho}$ of $\rho$ is the map defined by

$$
\begin{aligned}
\chi_{\rho}: G & \rightarrow \mathbb{C}, \\
\chi_{\rho}(g) & =\operatorname{Tr}(\rho(g)),
\end{aligned}
$$

where $\operatorname{Tr}(\alpha)$ denotes the trace of the linear map $\alpha$ (i.e., the trace of any matrix of $\alpha$ ). Note that $\chi_{\rho}(\mathrm{Id})=\operatorname{dim}(\rho)$ and that $\chi_{\rho}$ is a class function on $G$ (meaning that it is constant on each conjugacy class of $G$.)

The usefulness of characters lies in the following.

Fact. Two complex representations are isomorphic if and only if they have the same character; the set of complex irreducible characters is an orthonormal basis for the space of class functions in $\mathbb{C}[G]$.

If $\rho$ is any complex representation of $G$, its character satisfies $\chi_{\rho}\left(g^{-1}\right)=\overline{\chi_{\rho}(g)}$

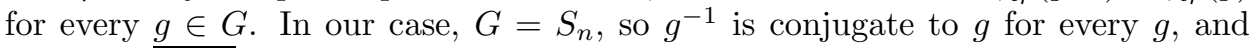
therefore $\overline{\chi_{\rho}(g)}=\chi_{\rho}(g)$; i.e., all the characters are real-valued. The irreducible characters of $S_{n}$ are therefore an orthonormal basis for the space of class functions on $\mathbb{R}\left[S_{n}\right]$. 
Given two representations $(\rho, V)$ and $\left(\rho^{\prime}, V^{\prime}\right)$ of $G$, we can form their direct sum, the representation $\left(\rho \oplus \rho^{\prime}, V \oplus V^{\prime}\right)$, and their tensor product, the representation $\left(\rho \otimes \rho^{\prime}, V \otimes V^{\prime}\right)$. We have $\chi_{\rho \oplus \rho^{\prime}}=\chi_{\rho}+\chi_{\rho^{\prime}}$, and $\chi_{\rho \otimes \rho^{\prime}}=\chi_{\rho} \cdot \chi_{\rho^{\prime}}$.

2.2. Fourier transforms and convolutions. In this section, we recall the basic notions of Fourier analysis on finite non-Abelian groups. For more background, see for example [28]. Noting that the normalization chosen differs in various texts, we set out our convention below.

Definition 2. Let $G$ be a finite group, and let $f, g: G \rightarrow \mathbb{R}$ be two real-valued functions on $G$. Their convolution $f * g$ is the real-valued function defined by

$$
f * g(t)=\frac{1}{|G|} \sum_{s \in G} f\left(t s^{-1}\right) g(s) \quad(t \in G) .
$$

Definition 3. The Fourier transform of a real-valued function $f: G \rightarrow \mathbb{R}$ is a matrix-valued function on irreducible representations; its value at the irreducible representation $\rho$ is the matrix

$$
\widehat{f}(\rho)=\frac{1}{|G|} \sum_{s \in G} f(s) \rho(s) .
$$

We now recall two related formulas we will need: the Fourier transform of a convolution, and the Fourier inversion formula. If $f, g: G \rightarrow \mathbb{R}$, and $\rho$ is an irreducible representation of $G$, then

$$
\widehat{f * g}(\rho)=\widehat{f}(\rho) \widehat{g}(\rho) .
$$

The Fourier transform is invertible; we have

$$
f(s)=\sum_{\rho \in R} \operatorname{dim}(\rho) \operatorname{Tr}\left[\widehat{f}(\rho) \rho\left(s^{-1}\right)\right] .
$$

In other words, the Fourier transform contains all the information about a function $f$.

2.3. Cayley graphs. Recall that if $G$ is a group, and $X \subset G$ is inverse-closed, the Cayley graph on $G$ generated by $X$ is the graph with vertex-set $G$ and edge-set $\left\{\{u, v\} \in G^{(2)}: u v^{-1} \in X\right\}$; it is sometimes denoted by $\operatorname{Cay}(G, X)$. In fact, we will only be considering cases where $G=S_{n}$ and $X$ is a union of conjugacy classes (i.e., $X$ is conjugation-invariant).

The relevance of this notion to our problem stems from the following observation. Consider the Cayley graph $\Gamma_{1}$ on $S_{n}$ generated by the set of fixed-point free permutations,

$$
\mathrm{FPF}=\left\{\sigma \in S_{n}: \sigma(i) \neq i \forall i \in[n]\right\} .
$$

As observed in section 1.1, a 1-intersecting family of permutations is precisely an independent set in this graph. More generally, a $k$-intersecting family of permutations is precisely an independent set in the Cayley graph $\Gamma_{k}$ on $S_{n}$ with generating set

$$
\mathrm{FPF}_{k}=\left\{\sigma \in S_{n}: \sigma \text { has at most } k \text { fixed points }\right\} \text {. }
$$

For any real matrix $A \in \mathbb{R}[G \times G]$, the left action of $A$ on $\mathbb{R}[G]$ is defined as follows:

$$
(A f)(\sigma)=\sum_{\tau \in G} A_{\sigma, \tau} f(\tau)
$$


Crucially, the adjacency matrix of a Cayley graph operates on functions in $\mathbb{R}[G]$ by convolution with the characteristic function of the generating set.

Theorem 9. Let $G$ be a finite group, let $X \subset G$ be inverse-closed, let $\operatorname{Cay}(G, X)$ be the Cayley graph on $G$ generated by $X$, and let $A$ be the adjacency matrix of $\operatorname{Cay}(G, X)$. Then for any function $f: G \rightarrow \mathbb{R}$,

$$
\text { Af }=|G|\left(1_{X} * f\right) \text {. }
$$

Proof. For any $f \in \mathbb{R}[G]$, and any $\sigma \in G$, we have

$$
(A f)(\sigma)=\sum_{\tau \in G} A_{\sigma, \tau} f(\tau)=\sum_{\tau \in G} 1_{X}\left(\sigma \tau^{-1}\right) f(\tau)=|G|\left(1_{X} * f\right)(\sigma),
$$

as required.

Taking the Fourier transform of both sides of (5), we obtain

$$
\widehat{A f}=|G| \cdot \widehat{1_{X}} \widehat{f} .
$$

If $X$ is conjugation-invariant, as in our case, then it follows from Schur's Lemma that $\widehat{1_{X}}(\rho)$ is a scalar matrix for any complex irreducible representation $\rho$. It is well known that for any finite group $G$, the entries of the matrices of a complete set of complex irreducible representations of $G$ form an orthogonal basis for the space of all complex-valued functions on $G$. So in our case, these entries form a complete set of eigenfunctions of $A$. This implies the following.

Theorem 10 (Babai; Diaconis-Shahshahani; Roichman [3, 7, 26]). Let $G$ be a finite group, and let $R$ be a complete set of complex irreducible matrix representations of $G$, as above. Let $X \subset G$ be inverse-closed and conjugation-invariant, and let Cay $(G, X)$ be the Cayley graph on $G$ with generating set $X$. Let $A$ be the adjacency matrix of $\operatorname{Cay}(G, X)$. For any $\rho \in R$, and any $i, j \leq \operatorname{dim}(\rho)$, consider the function $\rho_{i j}(\sigma)=\rho(\sigma)_{i j}$. Then $\left\{\rho_{i j}\right\}_{\rho, i, j}$ is a complete set of eigenvectors of A. Furthermore, the eigenvalue of $\rho_{i j}$ is

$$
\lambda_{\rho}=\frac{1}{\operatorname{dim}(\rho)} \sum_{\tau \in X} \chi_{\rho}(\tau)=\frac{|G|\left\langle\chi_{\rho}, 1_{X}\right\rangle}{\operatorname{dim}(\rho)}
$$

which depends only on $\rho$.

2.4. Hoffman's bound. First, we need a word regarding normalization, as this is always a potential source of confusion when doing Fourier analysis. Given a graph $G$, we use the uniform probability measure on the vertex-set $V$ of $G$, not the counting measure. The uniform measure induces the following inner product on $\mathbb{R}[V]:$

$$
\langle f, g\rangle=\frac{1}{|V|} \sum_{v \in V} f(v) g(v)
$$

this induces the Euclidean norm

$$
\|f\|_{2}=\sqrt{\langle f, f\rangle} .
$$

If $G=(V, E)$ is a graph, the adjacency matrix $A$ of $G$ is defined by

$$
A_{v, w}=1_{\{v w \in E(G)\}} \quad(v, w \in V(G)) .
$$

This is a real, symmetric, $n \times n$ matrix, so there exists an orthonormal system of $n$ eigenvectors of $A$, which forms a basis for $\mathbb{R}[V]$. (Note that the eigenvalues of $A$ are often referred to as the eigenvalues of $G$.) 
Hoffman [17 observed the following useful bound on the measure of an independent set in a regular graph, in terms of the eigenvalues of the graph:

Theorem 11 ([17]). Let $G=(V, E)$ be a d-regular, $n$-vertex graph. Let $A$ be the adjacency matrix of $G$. Let $\left\{v_{1}, v_{2}, \ldots, v_{n}\right\}$ be an orthonormal system of eigenvectors of $A$, with corresponding eigenvalues $d=\lambda_{1} \geq \lambda_{2} \geq \ldots \geq \lambda_{n}=\lambda_{\min }$ (so that $v_{1}$ is the all-1's vector). If $I \subset V$ is an independent set in $G$, then

$$
\frac{|I|}{|V|} \leq \frac{-\lambda_{\min }}{\lambda_{1}-\lambda_{\min }}
$$

If equality holds, then

$$
1_{I} \in \operatorname{Span}\left(\left\{v_{1}\right\} \cup\left\{v_{i}: \lambda_{i}=\lambda_{\min }\right\}\right) .
$$

Proof. Let $f=1_{I}$, and let $\alpha=\frac{|I|}{|V|}$. Observe that

$$
f^{t} A f=\sum_{v, w \in I} A_{v, w}=2 e(G[I])=0,
$$

since $I$ is independent. Write $f$ as a linear combination of the eigenvectors:

$$
f=\sum_{i=1}^{n} a_{i} v_{i} .
$$

Then $\alpha=\left\langle f, v_{1}\right\rangle=a_{1}$. Moreover, by Parseval's identity, we have $\sum_{i} a_{i}^{2}=\|f\|_{2}^{2}=$ $\alpha$. Now,

$$
0=f^{t} A f=\sum_{i=1}^{n} a_{i}^{2} \lambda_{i} \geq a_{1}^{2} \lambda_{1}+\sum_{i=2}^{n} a_{i}^{2} \lambda_{\min }=\alpha^{2} \lambda_{1}+\left(\alpha-\alpha^{2}\right) \lambda_{\min } .
$$

Rearranging gives (8). If equality holds, then $a_{i} \neq 0$ implies that $i=1$ or $\lambda_{i}=\lambda_{\min }$, completing the proof.

A variant of Hoffman's theorem, which will be crucial for us, comes from weighting the edges of the graph $G$ with real (possibly negative) weights.

Theorem 12. Let $G=(V, E)$ be an n-vertex graph. Let $G_{1}, \ldots, G_{t}$ be regular, spanning subgraphs of $G$, all having $\left\{v_{1}, v_{2}, \ldots, v_{n}\right\}$ as an orthonormal system of eigenvectors (where $v_{1}$ is the all-1's vector). Let $\lambda_{i}^{(j)}$ be the eigenvalue of $v_{i}$ in $G_{j}$. Let $\beta_{1}, \ldots, \beta_{t} \in \mathbb{R}$, and let $\lambda_{i}=\sum_{j} \beta_{j} \lambda_{i}^{(j)}$, and let $\lambda_{\min }=\min _{i} \lambda_{i}$. If $I \subset V$ is an independent set in $G$, then

$$
\frac{|I|}{|V|} \leq \frac{-\lambda_{\min }}{\lambda_{1}-\lambda_{\min }}
$$

If equality holds, then

$$
1_{I} \in \operatorname{Span}\left(\left\{v_{1}\right\} \cup\left\{v_{i}: \lambda_{i}=\lambda_{\min }\right\}\right) .
$$

Proof. The proof is a simple generalization of that of Theorem 11] For each $j$, let $A_{j}$ be the adjacency matrix of $G_{j}$, and let $A=\sum_{j} \beta_{j} A_{j}$. We have

$$
\begin{aligned}
0 & =f^{t} A f=\sum_{j=1}^{t} \beta_{j} f^{t} A_{j} f=\sum_{j=1}^{t} \beta_{j} \sum_{i=1}^{n} a_{i}^{2} \lambda_{i}^{(j)}=\sum_{i=1}^{n} a_{i}^{2} \lambda_{i} \\
& \geq \lambda_{1} a_{1}^{2}+\sum_{i} a_{i}^{2} \lambda_{\min }=\alpha^{2} \lambda_{1}+\left(\alpha-\alpha^{2}\right) \lambda_{\min } .
\end{aligned}
$$


Rearranging gives (9). If equality holds, then $a_{i} \neq 0$ implies that $i=1$ or $\lambda_{i}=\lambda_{\min }$, completing the proof.

We may think of the $\lambda_{i}$ 's above as the eigenvalues of the linear combination of graphs

$$
Y=\sum_{j=1}^{t} \beta_{j} G_{j} .
$$

The corresponding linear combination of adjacency matrices

$$
A=\sum_{j=1}^{t} \beta_{j} A_{j}
$$

is a so-called pseudo-adjacency matrix for $G$, meaning a symmetric matrix such that $A_{v, w}=0$ whenever $v w \notin E(G)$; the $\lambda_{i}$ 's are the eigenvalues of $A$.

Finally, we will need the following cross-independent version of Hoffman's Theorem. Variants of this theorem can be found in several sources, e.g. 2].

Theorem 13. Let $G=(V, E)$ be a d-regular, $n$-vertex graph, and let $\left\{v_{1}, v_{2}, \ldots, v_{n}\right\}$ be an orthonormal system of eigenvectors of $G$, with corresponding eigenvalues $d=\lambda_{1}, \lambda_{2}, \ldots, \lambda_{n}$ ordered by descending absolute value (so that $v_{1}$ is again the all-1's vector). Let $I, J \subset V$ be (not necessarily disjoint) sets of vertices in $G$ such that there are no edges of $G$ between $I$ and $J$. Then

$$
\sqrt{\frac{|I|}{|V|} \cdot \frac{|J|}{|V|}} \leq \frac{\left|\lambda_{2}\right|}{\lambda_{1}+\left|\lambda_{2}\right|}
$$

If equality holds, then

$$
1_{I}, 1_{J} \in \operatorname{Span}\left(\left\{v_{1}\right\} \cup\left\{v_{i}:\left|\lambda_{i}\right|=\left|\lambda_{2}\right|\right\}\right) .
$$

Proof. Let $f$ and $g$ be the characteristic functions of $I$ and $J$, respectively. As in the proof of Hoffman's Theorem, write

$$
f=\sum_{i=1}^{n} a_{i} v_{i}, g=\sum_{i=1}^{n} b_{i} v_{i} .
$$

Let $\alpha=\frac{|I|}{|V|}, \beta=\frac{|J|}{|V|}$. We have

$$
0=2 e(I, J)=f^{t} A g=\sum_{i=1}^{n} a_{i} b_{i} \lambda_{i}=\alpha \beta \lambda_{1}+\sum_{i=2}^{n} a_{i} b_{i} \lambda_{i} .
$$

Hence, by the Cauchy-Schwarz inequality,

$$
\alpha \beta \lambda_{1}=\left|\sum_{i=2}^{n} a_{i} b_{i} \lambda_{i}\right| \leq \sum_{i=2}^{n}\left|a_{i} b_{i} \lambda_{i}\right| \leq\left|\lambda_{2}\right| \sqrt{\alpha-\alpha^{2}} \sqrt{\beta-\beta^{2}} .
$$

Rearranging gives

$$
\sqrt{\frac{\alpha \beta}{(1-\alpha)(1-\beta)}} \leq \frac{\left|\lambda_{2}\right|}{\lambda_{1}} .
$$

Recall the AM-GM inequality for two real variables (see for example [15]):

$$
(\alpha+\beta) / 2 \geq \sqrt{\alpha \beta} .
$$


This implies that

$$
(1-\alpha)(1-\beta)=1-\alpha-\beta+\alpha \beta \leq 1-2 \sqrt{\alpha \beta}+\alpha \beta=(1-\sqrt{\alpha \beta})^{2},
$$

and therefore

$$
\sqrt{(1-\alpha)(1-\beta)} \leq 1-\sqrt{\alpha \beta}
$$

Hence,

Rearranging gives

$$
\frac{\sqrt{\alpha \beta}}{1-\sqrt{\alpha \beta}} \leq \frac{\left|\lambda_{2}\right|}{\lambda_{1}}
$$

$$
\sqrt{\alpha \beta} \leq \frac{\left|\lambda_{2}\right|}{\lambda_{1}+\left|\lambda_{2}\right|}
$$

as required. The case of equality is dealt with as in the original Hoffman theorem.

Note that the generalization of Hoffman's theorem that we mention above holds, with the obvious modifications, in the cross-independent case.

\section{Representation theory of $S_{n}$}

In this section we gather all the necessary background and results regarding the representation theory of $S_{n}$. Readers familiar with the basics of this theory are invited to skip the following subsection.

3.1. Basics. Our treatment follows Sagan [27.

A partition of $n$ is a nonincreasing sequence of integers summing to $n$, i.e. a sequence $\lambda=\left(\lambda_{1}, \ldots, \lambda_{k}\right)$ with $\lambda_{1} \geq \lambda_{2} \geq \ldots \geq \lambda_{k}$ and $\sum_{i=1}^{k} \lambda_{i}=n$; we write $\lambda \vdash n$. For example, $(3,2,2) \vdash 7$; we sometimes use the shorthand $(3,2,2)=\left(3,2^{2}\right)$. The following two orders on partitions of $n$ will be useful.

Definition 4 (Dominance order). Let $\lambda=\left(\lambda_{1}, \ldots, \lambda_{r}\right)$ and $\mu=\left(\mu_{1}, \ldots, \mu_{s}\right)$ be partitions of $n$. We say that $\lambda \unrhd \mu(\lambda$ dominates $\mu)$ if $\sum_{j=1}^{i} \lambda_{i} \geq \sum_{j=1}^{i} \mu_{i}$ for all $i$ (where we define $\lambda_{i}=0$ for all $i>r$, and $\mu_{j}=0$ for all $j>s$ ).

It is easy to see that this is a partial order.

Definition 5 (Lexicographic order). Let $\lambda=\left(\lambda_{1}, \ldots, \lambda_{r}\right)$ and $\mu=\left(\mu_{1}, \ldots, \mu_{s}\right)$ be partitions of $n$. We say that $\lambda>\mu$ if $\lambda_{j}>\mu_{j}$, where $j=\min \left\{i \in[n]: \lambda_{i} \neq \mu_{i}\right\}$.

It is easy to see that this is a total order which extends the dominance order.

The cycle-type of a permutation $\sigma \in S_{n}$ is the partition of $n$ obtained by expressing $\sigma$ as a product of disjoint cycles and listing its cycle lengths in nonincreasing order. The conjugacy classes of $S_{n}$ are precisely

$$
\left\{\sigma \in S_{n}: \text { cycle-type }(\sigma)=\lambda\right\}_{\lambda \vdash n} .
$$

Moreover, there is an explicit one-to-one correspondence between irreducible representations of $S_{n}$ (up to isomorphism) and partitions of $n$, which we now describe.

Let $\lambda=\left(\lambda_{1}, \ldots, \lambda_{k}\right)$ be a partiton of $n$. The Young diagram of $\lambda$ is an array of $n$ boxes, or cells, having $k$ left-justified rows, where row $i$ contains $\lambda_{i}$ cells. For example, the Young diagram of the partition $\left(3,2^{2}\right)$ is:

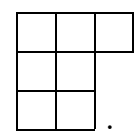


If the array contains the numbers $\{1,2, \ldots, n\}$ inside the cells, we call it a $\lambda$ tableau, or a tableau of shape $\lambda$; for example,

\begin{tabular}{|l|l|l|}
\hline 6 & 1 & 7 \\
\hline 5 & 4 & \multicolumn{1}{|l}{} \\
\cline { 1 - 2 } 3 & 2 & \multicolumn{1}{|c}{} \\
\cline { 1 - 1 } &
\end{tabular}

is a $\left(3,2^{2}\right)$-tableau. Two $\lambda$-tableaux are said to be row-equivalent if they have the same numbers in each row; if a $\lambda$-tableau $t$ has rows $R_{1}, \ldots, R_{l} \subset[n]$ and columns $C_{1}, \ldots, C_{l} \subset[n]$, then we let $R_{t}=S_{R_{1}} \times S_{R_{2}} \times \ldots \times S_{R_{l}}$ be the row-stablizer of $t$ and $C_{t}=S_{C_{1}} \times S_{C_{2}} \times \ldots \times S_{C_{k}}$ be the column-stabilizer.

A $\lambda$-tabloid is a $\lambda$-tableau with unordered row entries (or formally, a rowequivalence class of $\lambda$-tableaux); given a tableau $t$, we write $[t]$ for the tabloid it produces. For example, the $\left(3,2^{2}\right)$-tableau above produces the following $\left(3,2^{2}\right)$ tabloid:

$\left.\begin{array}{lll}\{1 & 6 & 7\end{array}\right\}$

Consider the natural permutation action of $S_{n}$ on the set $X^{\lambda}$ of all $\lambda$-tabloids; let $M^{\lambda}=\mathbb{R}\left[X^{\lambda}\right]$ be the corresponding permutation module, i.e. the real vector space with basis $X^{\lambda}$ and $S_{n}$ action given by extending the permutation action linearly. In general, $M^{\lambda}$ is reducible. However, we can describe a complete set of real irreducible representations, as follows.

If $t$ is a tableau, let $\kappa_{t}=\sum_{\pi \in C_{t}} \operatorname{sgn}(\pi) \pi$; this is an element of the group module $\mathbb{R}\left[S_{n}\right]$. Let $e_{t}=\kappa_{t}\{t\}$. This is a $( \pm 1)$-linear combination of tabloids, so is an element of $M^{\lambda}$; we call the $e_{t}$ 's polytabloids.

Definition 6. Let $\mu$ be a partition of $n$. The Specht module $S^{\mu}$ is the submodule of $M^{\mu}$ spanned by the $\mu$-polytabloids:

$$
S^{\mu}=\operatorname{Span}\left\{e_{t}: t \text { is a } \mu \text {-tabloid }\right\} .
$$

Theorem 14. The Specht modules are a complete set of pairwise inequivalent, irreducible representations of $S_{n}$.

Hence, any irreducible representation $\rho$ of $S_{n}$ is isomorphic to some $S^{\lambda}$; in this case, we say that $\rho$ has Young diagram $\lambda$. For example, $S^{(n)}=M^{(n)}$ is the trivial representation; $M^{\left(1^{n}\right)}$ is the left-regular representation, and $S^{\left(1^{n}\right)}$ is the sign representation $s g n$.

From now on we will write $[\lambda]$ for the equivalence class of the irreducible representation $S^{\lambda}, \chi_{\lambda}$ for the character $\chi_{S^{\lambda}}$, and $\xi_{\lambda}$ for the character of $M^{\lambda}$. Notice that the set of $\lambda$-tabloids forms a basis for $M^{\lambda}$, and therefore $\xi_{\lambda}(\sigma)$, the trace of the corresponding permutation representation, is precisely the number of $\lambda$-tableaux fixed by $\sigma$.

We now explain how the permutation modules $M^{\mu}$ decompose into irreducibles.

Definition 7. Let $\lambda, \mu$ be partitions of $n$. A $\lambda$-tableau is produced by placing a number between 1 and $n$ in each cell of the Young diagram of $\lambda$; if it has $\mu_{i}$ $i$ 's $(1 \leq i \leq n)$ it is said to have content $\mu$. A generalized $\lambda$-tableau is said to be semistandard if the numbers are nondecreasing along each row and strictly increasing down each column. 
Definition 8. Let $\lambda, \mu$ be partitions of $n$. The Kostka number $K_{\lambda, \mu}$ is the number of semistandard generalized $\lambda$-tableaux with content $\mu$.

Theorem 15 (Young's rule). Let $\mu$ be a partition of $n$. Then the permutation module $M^{\mu}$ decomposes as

$$
M^{\mu} \cong \bigoplus_{\lambda \vdash n} K_{\lambda, \mu} S^{\lambda}
$$

Hence,

$$
\xi_{\mu}=\sum_{\lambda \vdash n} K_{\lambda, \mu} \chi_{\lambda}
$$

For example, $M^{(n-1,1)}$, which corresponds to the natural permutation action of $S_{n}$ on $[n]$, decomposes as

$$
M^{(n-1,1)} \cong S^{(n-1,1)} \oplus S^{(n)},
$$

and therefore $\xi_{(n-1,1)}=\chi_{(n-1,1)}+1$.

The restriction of an irreducible representation of $S_{n}$ to the subgroup $\left\{\sigma \in S_{n}\right.$ : $\sigma(i)=i \forall i>n-k\}=S_{n-k}$ can be decomposed into irreducible representations of $S_{n-k}$ as follows:

Theorem 16 (The branching rule). Let $\alpha$ be a partition of $n-k$, and $\lambda$ a partition of $n$. We write $\alpha \subset^{k} \lambda$ if the Young diagram of $\alpha$ can be produced from that of $\lambda$ by sequentially removing $k$ corners (so that after removing the ith corner, we have the Young diagram of a partition of $n-i)$. Let $a_{\alpha, \lambda}$ be the number of ways of doing this; then we have

$$
[\lambda] \downarrow S_{n-k}=\sum_{\alpha \vdash n-k: \alpha \subset^{k} \lambda} a_{\alpha, \lambda}[\alpha]
$$

and therefore

$$
\chi_{\lambda} \downarrow S_{n-k}=\sum_{\alpha \vdash n-k: \alpha \subset^{k} \lambda} a_{\alpha, \lambda} \chi_{\alpha} .
$$

Definition 9. Let $\lambda=\left(\lambda_{1}, \ldots, \lambda_{k}\right)$ be a partition of $n$; if its Young diagram has columns of lengths $\lambda_{1}^{\prime} \geq \lambda_{2}^{\prime} \geq \ldots \geq \lambda_{l}^{\prime} \geq 1$, then the partition $\lambda^{t}=\left(\lambda_{1}^{\prime}, \ldots, \lambda_{l}^{\prime}\right)$ is called the transpose of $\lambda$, as its Young diagram is the transpose of that of $\lambda$.

Theorem 17. Let $\lambda$ be a partition of $[n]$; then $[\lambda] \otimes\left[1^{n}\right]=\left[\lambda^{t}\right]$. Hence, $\chi_{\lambda^{t}}=$ $\chi_{\lambda} \cdot \operatorname{sgn}$, and $\operatorname{dim}[\lambda]=\operatorname{dim}\left[\lambda^{t}\right]$.

Definition 10. The hook of a cell $(i, j)$ in the Young diagram of a partition $\mu$ is $H_{i, j}=\left\{\left(i, j^{\prime}\right): j^{\prime} \geq j\right\} \cup\left\{\left(i^{\prime}, j\right): i^{\prime} \geq i\right\}$. The hook length of $(i, j)$ is $h_{i, j}=\left|H_{i, j}\right|$.

Theorem 18 (Frame, Robinson, Thrall [9]). If $\lambda$ is a partition of $n$ with hook lengths $\left(h_{i, j}\right)$, then

$$
\operatorname{dim}[\lambda]=\frac{n !}{\prod_{i, j} h_{i, j}} .
$$

3.2. Lemmas regarding representations and characters. In this subsection we state and prove several lemmas regarding representations of $S_{n}$ and their characters; these will be instrumental in proving our main theorem. 


\subsubsection{Dimensions of irreducible representations.}

Lemma 2. Let $k \in \mathbb{N}$. Then there exists $E_{k}>0$ depending on $k$ alone such that for any irreducible representation $[\lambda]$ of $S_{n}$ with all rows and columns of length greater than $n-k, \operatorname{dim}[\lambda] \geq E_{k} n^{k+1}$.

To prove this lemma, we need two simple claims dealing with irreducible representations with a relatively long row or column, and a separate result dealing with the rest.

Claim 1. Let $[\lambda]$ be an irreducible representation whose first row or column is of length $n-t$. Then

$$
\operatorname{dim}[\lambda] \geq\left(\begin{array}{l}
n \\
t
\end{array}\right) e^{-t}
$$

Proof. Note that if $\lambda$ has first column of length $n-t$, then $\lambda^{t}$ has first row of length $n-t$. Since $\operatorname{dim}[\lambda]=\operatorname{dim}\left[\lambda^{t}\right]$, we may assume that $\lambda$ has first row of length $n-t$.

By the hook formula (12), it suffices to prove that

$$
\prod_{i, j} h_{i, j} \leq t !(n-t) ! e^{t}
$$

Delete the first row $R_{1}$ of the Young diagram of $\lambda$; the resulting Young diagram $D$ corresponds to a partition of $t$, and therefore a representation of $S_{t}$, which has dimension

$$
\frac{t !}{\prod_{(i, j) \in D} h_{i, j}} \geq 1
$$

Hence,

$$
\prod_{(i, j) \in D} h_{i, j} \leq t !
$$

We now bound the product of the hook lengths of the cells in the first row; this is of the form

$$
\prod_{(i, j) \in R_{1}} h_{i, j}=\prod_{j=1}^{n-t}\left(j+c_{j}\right),
$$

where $\sum_{j=1}^{n-t} c_{j}=t$. Using the AM/GM inequality, we obtain

$$
\begin{aligned}
\prod_{j=1}^{n-t} \frac{j+c_{j}}{j} & =\prod_{j=1}^{n-t}\left(1+\frac{c_{j}}{j}\right) \\
& \leq\left(\sum_{j=1}^{n-t} \frac{1+\frac{c_{j}}{j}}{n-t}\right)^{n-t} \\
& \leq\left(\frac{n-t+\sum_{j=1}^{n-t} c_{j}}{n-t}\right)^{n-t} \\
& =\left(1+\frac{t}{n-t}\right)^{n-t} \\
& <e^{t}
\end{aligned}
$$


Hence,

$$
\prod_{i, j} h_{i, j} \leq t !(n-t) ! e^{t}
$$

as desired.

Note that, if $t$ is suffiently small depending on $n,\left(\begin{array}{l}n \\ t\end{array}\right) e^{-t}$ is an increasing function of $t$ :

Claim 2. Let $L(n, t)=\left(\begin{array}{l}n \\ t\end{array}\right) e^{-t}$. Then $L(n, t) \leq L(n, t+1)$ for all $t \leq(n-e) /(e+1)$.

Proof. Observe that

$$
\frac{L(n, t)}{L(n, t+1)}=\frac{e(t+1)}{n-t} .
$$

Solving for $t$ when this expression is at most 1 proves the claim.

For the representations not covered by Claim 1 we use the following.

Theorem 19 (24]). If $\alpha, \epsilon>0$, then there exists $N(\alpha, \epsilon) \in \mathbb{N}$ such that for all $n>N(\alpha, \epsilon)$, any irreducible representation $[\lambda]$ of $S_{n}$ which has all rows and columns of length at most $n / \alpha$ has

$$
\operatorname{dim}[\lambda] \geq(\alpha-\epsilon)^{n} .
$$

The proof of Lemma 2 is now immediate.

Proof of Lemma 2. If the partition $\lambda$ contains a row or column of length between $n-(n-e) /(e+1)$ and $n-k-1$, then by Claims 1 and 2 ,

$$
\operatorname{dim}[\lambda] \geq e^{-(k+1)}\left(\begin{array}{c}
n \\
k+1
\end{array}\right) \geq E_{k} n^{k+1},
$$

provided we choose $E_{k}>0$ sufficiently small. Otherwise, the conditions of Theorem 19 hold with $\alpha=(e+1) / e-\epsilon^{\prime}$ for some small $\epsilon^{\prime}>0$, and therefore, by Theorem 19

$$
\operatorname{dim}[\lambda] \geq n^{k+1},
$$

provided $n$ is sufficiently large depending on $k$, completing the proof.

3.2.2. Character tables and their minors. We will be working with certain minors of the character table of $S_{n}$. The following lemmas imply that certain related matrices are upper triangular with 1's all along the diagonal.

Lemma 3. If $\lambda, \mu$ are partitions of $n$, let $K_{\lambda, \mu}$ denote the Kostka number, the number of semistandard $\lambda$-tableaux of content $\mu$. If $K_{\lambda, \mu} \geq 1$, then $\lambda \geq \mu$. Moreover, $K_{\lambda, \lambda}=1$ for all $\lambda$.

Proof. See for example [27], Proposition 4.4.3.

Lemma 4. Let $\lambda$ be a partition of $n$, and let $\xi_{\lambda}$ be the character of the permutation module $M^{\lambda}$. Let $\sigma \in S_{n}$. If $\xi_{\lambda}(\sigma) \neq 0$, then cycle-type $(\sigma) \unlhd \lambda$. Moreover, if cycle-type $(\sigma)=\lambda$, then $\xi_{\lambda}(\sigma)=1$.

Proof. The set of $\lambda$-tabloids is a basis for the permutation module $M^{\lambda}$. Thus, $\xi_{\lambda}(\sigma)$, which is the trace of the corresponding representation on the permutation $\sigma$, is simply the number of $\lambda$-tabloids fixed by $\sigma$. If $\xi_{\lambda}(\sigma) \neq 0$, then $\sigma$ fixes some $\lambda$-tabloid $[t]$. Hence, every row of length $l$ in $[t]$ is a union of the sets of numbers in a collection of disjoint cycles of total length $l$ in $\sigma$. Thus, the cycle-type of $\sigma$ is a refinement of $\lambda$, and therefore cycle-type $(\sigma) \unlhd \lambda$, as required. If $\sigma$ has cycle-type 
$\lambda$, then it fixes just one $\lambda$-tabloid, the one whose rows correspond to the cycles of $\sigma$, so $\xi_{\lambda}(\sigma)=1$.

Theorem 20. Let $C$ be the character table of $S_{n}$, with rows and columns indexed by partitions / conjugacy classes in decreasing lexicographic order (so $C_{\lambda, \mu}=$ $\chi_{\lambda}\left(\sigma_{\mu}\right)$, where $\sigma_{\mu}$ is a permutation with cycle-type $\mu$, and the top-left corner of $C$ is $\left.\chi_{(n)}\left(\sigma_{(n)}\right)\right)$. Then the contiguous square minor $\tilde{C}$ of $C$ with rows and columns $\psi: \psi>\left(n-k, 1^{k}\right)$ is invertible and does not depend on $n$, provided $n>2 k$.

Proof. Let $K$ be the Kostka matrix, and let $D$ be the matrix of permutation characters,

$$
D_{\lambda, \mu}=\xi_{\lambda}\left(\sigma_{\mu}\right),
$$

where $\sigma_{\mu}$ denotes a permutation with cycle-type $\mu$. Let $\tilde{K}$ and $\tilde{D}$ denote the top-left minor of $K$ and $D$ respectively (i.e., the minor with rows and columns $\left.\psi: \psi>\left(n-k, 1^{k}\right)\right)$.

Recall that by Young's rule (Theorem 15), we have

$$
M^{\mu} \cong \bigoplus_{\lambda} K_{\lambda, \mu} S^{\lambda}
$$

and therefore

$$
\xi_{\mu}=\sum_{\lambda} K_{\lambda, \mu} \chi_{\lambda}
$$

Hence,

$$
\left(K^{t} C\right)_{\lambda, \mu}=\sum_{\tau} K_{\tau, \lambda} C_{\tau, \mu}=\sum_{\tau} K_{\tau, \lambda} \chi_{\tau}\left(\sigma_{\mu}\right)=\xi_{\lambda}\left(\sigma_{\mu}\right)=D_{\lambda, \mu},
$$

and therefore

$$
K^{t} C=D .
$$

Since the rows and columns of $K$ are sorted in decreasing lexicographic order, $K$ is upper triangular with 1's all along the diagonal, by Lemma 3, Therefore, $K^{t}$ is lower triangular with 1's all along the diagonal.

Since $K^{t}$ is lower triangular, in addition to (18), we also have

$$
\tilde{K}^{t} \tilde{C}=\tilde{D} \text {. }
$$

Since $\tilde{K}^{t}$ is lower triangular with 1's all along the diagonal, it is invertible, and therefore

$$
\tilde{C}=\left(\tilde{K}^{t}\right)^{-1} \tilde{D} .
$$

By Lemma 4, $\tilde{D}$ is upper triangular with 1's all along the diagonal, and is therefore invertible. It follows that $\tilde{C}$ is invertible also.

We will now show that $\tilde{K}$ and $\tilde{D}$ are independent of $n$, provided $n>2 k$; this will prove that $\tilde{C}$ is also independent of $n$.

Let $\lambda>\left(n-k, 1^{k}\right)$ be a partition. Then $\lambda_{1} \geq n-k$. Write $\lambda^{\prime}=\left(\lambda_{1}-\right.$ $\left.(n-k), \lambda_{2}, \ldots\right)$. (Note that this may not be a bona fide partition, as it may not be in nonincreasing order.) Now the mapping $\lambda \mapsto \lambda^{\prime}$ has the same image over $\left\{\lambda: \lambda>\left(n-k, 1^{k}\right)\right\}$ for all $n \geq 2 k$ : namely, 'partitions' of $k$ where the first row is not necessarily the longest.

We first consider $K$. Recall once again that $K_{\lambda, \mu}$ is the number of semistandard $\lambda$-tableaux of content $\mu$. Let $t$ be a semistandard $\lambda$-tableau of content $\mu$; we now 
count the number of choices for $t$. Since the numbers in a semistandard tableau are strictly increasing down each column and nonincreasing along each row, and $\mu_{1} \geq n-k$, we must always place 1's in the first $n-k$ cells of the first row of $t$. We must now fill the rest of the cells with content $\mu^{\prime}$. Provided $n \geq 2 k$, $\mu^{\prime}$ is independent of $n$, and the remaining cells in the first row have no cells below them, so the number of ways of doing this is independent of $n$. Hence, the entire minor $\tilde{K}$ is independent of $n$.

Now consider $D$. Recall that $D_{\lambda, \mu}=\xi_{\lambda}\left(\sigma_{\mu}\right)$ is simply the number of $\lambda$-tabloids fixed by $\sigma_{\mu}$. To count these, first note that the numbers in the long cycle of $\sigma$ (which has length at least $n-k$ ) must all be in the first row of the $\lambda$-tabloid (otherwise the long cycle of $\sigma$ must intersect two or more rows, as $n-k>k$.) This leaves us with a $\left(\lambda_{1}-\mu_{1}, \lambda_{2}, \ldots, \lambda_{r}\right)$-'tableau', which we need to fill with the remaining $n-\mu_{1}$ elements in such a way that $\sigma$ fixes it. It is easy to see that, again, the number of ways of doing this is independent of $n$.

In particular, if $n \geq 2 k$, the number of partitions $\lambda$ of $n$ such that $\lambda \geq\left(n-k, 1^{k}\right)$ is independent of $n$; we denote it by $q_{k}$. Note that

$$
q_{k}=\sum_{t=0}^{k} p_{t},
$$

where $p_{t}$ denotes the number of partitions of $t$.

We need a slightly more general result, which allows us to split some of the partitions.

Definition 11. Assume that $n>3 k+1$, and let $\mu=\left(\mu_{1}, \ldots, \mu_{r}\right)$ be a partition of $n$ with $\mu_{1} \geq n-k$. We define

$$
\operatorname{Split}(\mu)=\left(\mu_{1}-k-1, k+1, \mu_{2}, \ldots, \mu_{r}\right) .
$$

It is easy to see that $\operatorname{Split}(\mu)$ is indeed a partition (i.e., it is in descending order). Further, exactly one of $\mu$ and $\operatorname{Split}(\mu)$ is even.

Theorem 21. Let $C$ be as above, and let $n>3 k+1$. Let $\phi_{1}, \ldots, \phi_{q_{k}-1}$ be the partitions $>\left(n-k, 1^{k}\right)$. Let $\mu_{1}, \ldots, \mu_{q_{k}-1}$ be partitions such that, for each $j$, either $\mu_{j}=\phi_{j}$ or else $\mu_{j}=\operatorname{Split}\left(\phi_{j}\right)$. Then the square minor $\breve{C}$ of $C$ with ith row $\phi_{i}$ and jth column $\mu_{j}$ is independent of the choices of the $\mu_{j}$ 's, so is always equal to the top-left minor $\tilde{C}$.

Proof. It is easy to see that if $\lambda, \mu>\left(n-k, 1^{k}\right), \sigma$ has cycle-type $\mu$ and $\sigma^{\prime}$ has cycle-type $\operatorname{Split}(\mu)$, then in fact, $\xi_{\lambda}(\sigma)=\xi_{\lambda}\left(\sigma^{\prime}\right)$. (All rows of a $\lambda$-tabloid below the first have length at most $k$. Hence, if a permutation $\sigma^{\prime}$ with cycle-type $\operatorname{Split}(\mu)$ fixes a $\lambda$-tabloid $[t]$, the numbers in the $\left(\mu_{1}-k-1\right)$-cycle and the $(k+1)$-cycle must all lie in the first row of $[t]$. It follows that a permutation $\sigma$ produced by merging these two cycles of $\sigma^{\prime}$ fixes exactly the same $\lambda$-tabloids as $\sigma^{\prime}$ does.) Since the Kostka matrix $K$ is upper triangular with 1's all down the diagonal, $\left\{\xi_{\lambda}: \lambda>\left(n-k, 1^{k}\right)\right\}$ and $\left\{\chi_{\lambda}: \lambda>\left(n-k, 1^{k}\right)\right\}$ are bases for the same linear space, and therefore

$$
\chi_{\lambda}(\sigma)=\chi_{\lambda}\left(\sigma^{\prime}\right)
$$

for each $\lambda>\left(n-k, 1^{k}\right)$. Thus,

$$
C_{\lambda, \operatorname{Split}(\mu)}=C_{\lambda, \mu} \quad \forall \lambda, \mu>\left(n-k, 1^{k}\right)
$$

as required. 
3.2.3. Functions with Fourier transform concentrated on the 'fat' irreducible representations. One of the recurring themes in applications of discrete Fourier analysis to combinatorics is proving that certain functions depend on few coordinates, by showing that their Fourier transform is concentrated on the 'low frequencies', the characters indexed by small sets. Examples of this can be found for example in [5], 11] and [12. In this paper, we need a non-Abelian analogue. We show that functions on $S_{n}$ whose Fourier transform is supported on irreducible representations which are large with respect to the lexicographic order are spanned by the cosets of the pointwise stabilizers of small sets.

Definition 12. Let $V_{k}$ be the linear space of functions whose Fourier transform is supported only on representations $\geq\left(n-k, 1^{k}\right)$.

We are now ready to prove

Theorem 7, $V_{k}$ is the span of the $k$-cosets.

Proof. First, we show that the characteristic function of any $k$-coset is indeed in $V_{k}$. Let $T=T_{a_{1} \mapsto b_{1}, \ldots, a_{k} \mapsto b_{k}}$. It is easy to check that if $f: S_{n} \rightarrow \mathbb{R}, \tau, \pi \in S_{n}$, and

$$
\begin{aligned}
g: S_{n} & \rightarrow \mathbb{R} \\
\sigma & \mapsto f(\pi \sigma \tau),
\end{aligned}
$$

i.e. the function $g$ is a 'double-translate' of $f$, then $\hat{f}(\rho)=0 \Rightarrow \hat{g}(\rho)=0$. Hence, if $f \in V_{k}$, so is $g$. (This says that $V_{k}$ is a 'two-sided ideal' of the group algebra $\mathbb{R}\left[S_{n}\right]$.)

Hence, by double-translation, without loss of generality, we may assume that $a_{i}=b_{i}=i$ for each $i \in[k]$, i.e. $T=T_{1 \mapsto 1, \ldots, k \mapsto k}$, so $T \cong S_{n-k}$. We use the branching rule (Theorem [16). If $\rho<\left(n-k, 1^{k}\right)$, then $\rho$ has at least $k+1$ cells outside the first row. In that case, every $\mu \subset^{k} \rho$ is a nontrivial irreducible representation of $S_{n-k}$. We claim that this implies that

$$
\sum_{\sigma \in S_{n-k}} \mu(\sigma)=0
$$

To see this, observe that the linear map $\sum_{\sigma \in S_{n-k}} \mu(\sigma)$ commutes with $\mu$, and therefore by Schur's Lemma, it must be a scalar multiple of the identity map. Its trace is zero, since $\chi_{\mu}$ is orthogonal to the trivial character, so it must be the zero map. Hence, $\widehat{1_{T}}(\rho)=0$ for each $\rho<\left(n-k, 1^{k}\right)$.

(Note also that if $\rho=\left(n-k, 1^{k}\right)$, then the only $\mu \subset^{k} \rho$ with nonzero sum on $T$ is that corresponding to the partition $(n-k)$, i.e. the trivial representation, which has sum $(n-k)$ ! on $T$. Therefore, $\widehat{1_{T}}\left(\left(n-k, 1^{k}\right)\right) \neq 0$. This will be useful in the proof of Theorem 22,

In the other direction, let $f \in V_{k}$. Using the Fourier inversion formula, we have

$$
f(\sigma)=\sum_{\rho \geq\left(n-k, 1^{k}\right)} \operatorname{dim}[\rho] \operatorname{Tr}\left[\hat{f}(\rho) \rho\left(\sigma^{-1}\right)\right] .
$$

Consider the permutation module $M^{\left(n-k, 1^{k}\right)}$. By Young's Rule (Theorem [15), $M^{\left(n-k, 1^{k}\right)}$ must contain at least one copy of every Schur module $S^{\rho}$ with $\rho \geq$ $\left(n-k, 1^{k}\right)$, and no others. We can therefore rewrite the previous formula as

$$
f(\sigma)=\operatorname{Tr}\left[A \psi\left(\sigma^{-1}\right)\right],
$$


where $\psi$ is a matrix representation corresponding to $M^{\left(n-k, 1^{k}\right)}$, and $A$ is a blockdiagonal matrix whose blocks correspond to the irreducible modules $S^{\rho}$; i.e., it has $K_{\rho,\left(n-k, 1^{k}\right)}$ blocks corresponding to $\rho$, all equal to

$$
\frac{\operatorname{dim}[\rho]}{K_{\rho,\left(n-k, 1^{k}\right)}} \hat{f}(\rho)
$$

where the Kostka number $K_{\rho,\left(n-k, 1^{k}\right)}$ is the multiplicity of $S^{\rho}$ in $M^{\left(n-k, 1^{k}\right)}$.

Next, recall that the permutation module $M^{\left(n-k, 1^{k}\right)}$ corresponds to the permutation action of $S_{n}$ on $\left(n-k, 1^{k}\right)$-tabloids, which can be identified with ordered $k$-tuples of distinct numbers between 1 and $n$. Since trace is conjugation-invariant, we can perform a change of basis, replacing $A$ and $\psi$ by similar matrices $B$ and $\phi$, so that

$$
f(\sigma)=\operatorname{Tr}\left[B \phi\left(\sigma^{-1}\right)\right],
$$

where $\phi_{\alpha, \beta}(\tau)$ is 1 if $\tau$ takes the ordered $k$-tuple $\alpha$ to the ordered $k$-tuple $\beta$, and 0 otherwise. But then equation (22) means precisely that

$$
f=\sum_{\alpha, \beta} B_{\alpha, \beta} T_{\alpha \mapsto \beta},
$$

completing the proof.

\section{Proof of MAIN THEOREMS}

4.1. Proof strategy. In this section we finally proceed to eat the pudding. In view of Theorem 7, we would like to prove that whenever $I$ is a maximum-sized $k$ intersecting family, the Fourier transform of its characteristic function is supported on the irreducible representations corresponding to partitions $\rho \geq\left(n-k, 1^{k}\right)$, i.e. those whose Young diagram has first row of length at least $n-k$. Let us call these representations the 'fat' representations, and their transposes (those whose Young diagram has first column of height at least $n-k$ ) the 'tall' representations. The rest will be called 'medium' representations.

Given a $k$-intersecting family $I \subset S_{n}$, we will first consider it as an independent set in the Cayley graph $\Gamma_{k}$ on $S_{n}$ generated by $\mathrm{FPF}_{k}$, the set of permutations with less than $k$ fixed points. Since $\mathrm{FPF}_{k}$ is a union of conjugacy classes, by Theorems 10 and 14, the eigenvalues of $\Gamma_{k}$ are given by

$$
\lambda_{\rho}^{(k)}=\frac{1}{\operatorname{dim}[\rho]} \sum_{\sigma \in \mathrm{FPF}_{k}} \chi_{\rho}(\sigma) \quad(\rho \vdash n) .
$$

Fixed-point-free permutations are also called derangements, and $\Gamma_{1}$ is also called the derangement graph. Let $d_{n}=\left|\mathrm{FPF}_{1}(n)\right|$; it is well known (and easy to see, using the inclusion-exclusion formula) that

$$
d_{n}=\sum_{i=0}^{n}(-1)^{i}\left(\begin{array}{c}
n \\
i
\end{array}\right)(n-i) !=\sum_{i=0}^{n}(-1)^{i} \frac{n !}{i !}=(1 / e+o(1)) n ! .
$$

For $n \geq 5$, the eigenvalues of $\Gamma_{1}$ satisfy:

$$
\begin{aligned}
\lambda_{(n)}^{(1)} & =d_{n}, \\
\lambda_{(n-1,1)}^{(1)} & =-d_{n} /(n-1), \\
\left|\lambda_{\rho}^{(1)}\right| & <c d_{n} / n^{2}<d_{n} /(n-1) \quad \text { for all other } \rho \vdash n
\end{aligned}
$$


(where $c$ is an absolute constant). Hence, the matrix $\Gamma_{1}$ has

$$
\lambda_{\min } / \lambda_{1}=-1 /(n-1) .
$$

As observed in [14 and 25], this implies via Hoffman's bound (Theorem 11) that any 1-intersecting family $I \subset S_{n}$ satisfies $|I| \leq(n-1)$ !. If equality holds, then $1_{I} \in V_{1}$, from which we may conclude (e.g. from the $k=1$ case of Theorems 7 and 8) that $I$ is a 1 -coset. Also, we may conclude via Theorem 13 that any 1 -crossintersecting pair of families $I, J \subset S_{n}$ safisfy $|I||J| \leq((n-1) !)^{2}$. If equality holds, then $1_{I}, 1_{J} \in V_{1}$, which enables us to conclude that $I=J$ is a 1 -coset of $S_{n}$. This proves Leader's conjecture on 1-cross-intersecting families in $S_{n}$ for $n \geq 5$ (it can be verified directly for $n=4$ ).

However, for $k$ fixed and $n$ large, calculating the least eigenvalue of $\Gamma_{k}$ and applying Hoffman's bound only gives an upper bound of $\Theta((n-1) !)$ for the size of a $k$-intersecting subset of $S_{n}$. Indeed,

$$
\begin{aligned}
\lambda_{(n-1,1)}^{(k)} & =\frac{1}{\operatorname{dim}[n-1,1]} \sum_{\sigma \in \mathrm{FPF}_{k}} \chi_{(n-1,1)}(\sigma) \\
& =\frac{1}{n-1} \sum_{\sigma \in \mathrm{FPF}_{k}}\left(\xi_{(n-1,1)}(\sigma)-1\right) \\
& =\frac{1}{n-1} \sum_{i=0}^{k-1}\left(\begin{array}{c}
n \\
i
\end{array}\right) d_{n-i}(i-1) \\
& =-\frac{1}{n-1}(1 / e+o(1)) n !\left(1-\sum_{i=1}^{k-2} \frac{i}{(i+1) !}\right) .
\end{aligned}
$$

Note that

$$
\sum_{i=1}^{\infty} \frac{i}{(i+1) !}=\left.\frac{d}{d x} \frac{e^{x}-1}{x}\right|_{x=1}=1
$$

so for any $k \in \mathbb{N}$,

$$
1-\sum_{i=1}^{k-2} \frac{i}{(i+1) !}>0,
$$

and therefore $\lambda_{(n-1,1)}^{(k)}=-\Theta_{k}((n-1) !)$. It turns out that $\left|\lambda_{\rho}^{(k)}\right| \leq a_{k} n ! / n^{2}$ for all partitions $\rho \neq(n),(n-1,1)$, where $a_{k}>0$ depends on $k$ alone. Hence, if $k$ is fixed and $n$ is large, $\Gamma_{k}$ has

$$
\lambda_{\min }=\lambda_{(n-1,1)}^{(k)}=-\Theta_{k}((n-1) !) .
$$

Hence, applying Hoffman's bound only gives

$$
|I| \leq \Theta_{k}((n-1) !)
$$

for a $k$-intersecting $I \subset S_{n}$.

Instead, we will construct a linear combination $Y$ of subgraphs of $\Gamma_{k}$ (each a Cayley graph generated by a conjugacy class within $\mathrm{FPF}_{k}$ ) which has the correct eigenvalues for us to prove Theorems 5 and 6 from Theorem [12, By Theorem 10 if $X_{1}, \ldots, X_{t}$ are conjugacy classes within $\mathrm{FPF}_{k}$, and $\beta_{1}, \ldots, \beta_{t} \in \mathbb{R}$, then the 
eigenvalues of the linear combination

$$
Y=\sum_{j=1}^{t} d_{j} \operatorname{Cay}\left(S_{n}, X_{j}\right)
$$

are

$$
\lambda_{\rho}=\frac{1}{\operatorname{dim}[\rho]} \sum_{j=1}^{t} d_{j} \sum_{\sigma \in X_{j}} \chi_{\rho}(\sigma)=\frac{1}{\operatorname{dim}[\rho]} \sum_{j=1}^{t} d_{j}\left|X_{j}\right| \chi_{\rho}\left(\tau_{j}\right) \quad(\rho \vdash n),
$$

where $\tau_{j}$ denotes any permutation in $X_{j}$. So the eigenvalues of $Y$ still correspond to partitions of $n$ and are therefore relatively easy to handle.

The value of $\lambda_{\min } / \lambda_{1}$ required in Theorem 12 to produce the upper bound in Theorem 5 is as follows:

Corollary 1. Define

$$
\omega=\omega_{n, k}=-\frac{(n-k) !}{n !-(n-k) !}=-\frac{1}{n(n-1) \ldots(n-k+1)-1} .
$$

Assume the conditions of Theorem 12. If $\lambda_{m i n} / \lambda_{1}=\omega$, and $I$ is an independent set in $G$, then $|I| \leq(n-k)$ !.

Proof. This is immediate from Theorem 12.

Since rescaling the linear combination of graphs makes no difference to the above, our aim will be to construct a linear combination $Y$ with $\lambda_{1}=1$ and $\lambda_{\min }=\omega$. Since equality has to hold in Theorem 12 when the independent set is a $k$-coset, we know that for any $k$-coset $T$, we must have

$$
1_{T} \in \operatorname{Span}\left(\left\{v_{1}\right\} \cup\left\{v_{i}: \lambda_{i}=\omega\right\}\right) .
$$

By Theorem 7 it follows that we must have $\lambda_{\rho}=\omega$ for each fat partition $\rho \neq(n)$.

We will in fact construct two linear combinations, $Y_{\text {even }}$ and $Y_{\text {odd }}$, from Cayley graphs generated by respectively even/odd conjugacy classes within $\mathrm{FPF}_{k}$. We design these linear combinations so that $\lambda_{(n)}=1$ and $\lambda_{\rho}=\omega$ for all fat $\rho \neq(n)$. Recalling for any partition $\rho$ that we have $\chi_{\rho^{t}}=\chi_{\rho} \cdot s g n$, where $s g n$ is the sign character, we see from (24) that for any partition $\rho$, we have

- $\lambda_{\rho^{t}}=\lambda_{\rho}$ in $Y_{\text {even }}$, whereas

- $\lambda_{\rho^{t}}=-\lambda_{\rho}$ in $Y_{\text {odd }}$.

Hence, simply taking

$$
Y=\frac{1}{2}\left(Y_{\text {even }}+Y_{\text {odd }}\right)
$$

will ensure that the eigenvalues of $Y$ corresponding to the tall representations are all zero.

Moreover, we will show that in both $Y_{\text {even }}$ and $Y_{\text {odd }}$ (and consequently in $Y$ ), the eigenvalues corresponding to the medium representations all have absolute value $|\lambda| \leq c_{k}|\omega| / \sqrt{n}=o(|\omega|)$, where $c_{k}>0$ depends on $k$ alone. So provided $n$ is sufficiently large, $\omega$ is both the minimal eigenvalue of $Y$ and the second largest in absolute value and is attained only on the nontrivial fat irreducible representations. The situation is summarized in Table 1 note that the $o(|\omega|)$ function is always the same function.

Applying Theorem 12 to $Y$ will not only prove that $|I| \leq(n-k)$ !, but also that if equality holds, then the Fourier transform of the characteristic function of $I$ is totally supported on the fat representations, yielding the proof of our main 
TABLE 1. Eigenvalues

\begin{tabular}{|c|c|c|c|c|c|}
\hline & $(n)$ & fat, $\neq(n)$ & $\left(1^{n}\right)$ & tall, $\neq\left(1^{n}\right)$ & medium \\
\hline$Y_{\text {even }}$ & 1 & $\omega$ & 1 & $\omega$ & $o(|\omega|)$ \\
\hline$Y_{\text {odd }}$ & 1 & $\omega$ & -1 & $-\omega$ & $o(|\omega|)$ \\
\hline$Y$ & 1 & $\omega$ & 0 & 0 & $o(|\omega|)$ \\
\hline
\end{tabular}

theorem (pending the results of section 5). Since $\omega$ is also the eigenvalue of secondlargest absolute value, we will also be able to deduce Theorem 4, concerning $k$ cross-intersecting families.

In order to carry out our plan, we will identify appropriate conjugacy classes $X_{j}$ to use as the generating sets for our Cayley graphs, and appropriate coefficients $d_{j}$. The set of linear equations the $d_{j}$ 's must satisfy will always correspond to a specific square minor of the character table of $S_{n}$, which turns out to be nonsingular and independent of $n$ (for $n$ sufficiently large). The latter statement is precisely the content of Theorems 20 and 21.

As for the medium representations, we will show that their eigenvalues have small absolute value using the lower bound we proved on their dimensions in Lemma 2.

4.2. Calculations of eigenvalues of Cayley graphs. The Cayley graphs we will use to construct our linear combination will be generated by conjugacy classes of permutations with specific cycle-types. These cycle-types will fall into two categories: those corresponding to partitions $>\left(n-k, 1^{k}\right)$, and those corresponding to partitions $\pi$, where

$$
(n-k-1, k+1) \geq \pi>\left(n-2 k-1, k+1,1^{k}\right) .
$$

Note that the second category is exactly the split of the first category (see definition [11). We need to use the split partitions in order to ensure that our conjugacy classes have the correct sign.

We will need the following bounds on the sizes of the above conjugacy classes:

Lemma 5. Let $X$ be a conjugacy class of $S_{n}$ with a cycle of length $n-t$, where $t<n / 2$. Then

$$
\frac{n !}{(n-t) t^{t}} \leq|X| \leq 2(n-1) !
$$

Proof. Suppose the cycle-type of $X$ is $\left(n-t, c_{1}, \ldots, c_{l}\right)$, where $l \leq t$ and $\sum_{i=1}^{l} c_{i}=t$. Define a mapping

$$
F: S_{n} \rightarrow X
$$

by taking a permutation $\sigma \in S_{n}$, writing it in sequence notation

$$
\sigma(1), \sigma(2), \ldots, \sigma(n),
$$

and then placing parentheses at the appropriate points, producing a permutation in $X$ (written in disjoint cycle notation). This mapping is clearly surjective, and each element of $X$ has the same number of preimages, $N$ say. Note that the preimages of a permutation in $X$ are obtained by rotating each cycle and then permuting the cycles of length $j$ for each $j$. Let $a_{j}=\left|\left\{i: c_{i}=j\right\}\right|$ for each $j$; then

$$
N=(n-t) \prod_{j} a_{j} ! \prod_{i=1}^{l} c_{i} .
$$


Observe that

$$
n / 2<n-t \leq N \leq(n-t) l ! \prod_{i=1}^{l} c_{i} \leq(n-t) l !(t / l)^{l} \leq(n-t) t^{l} \leq(n-t) t^{t},
$$

using the AM/GM inequality. Hence,

as required.

$$
\frac{n !}{(n-t) t^{t}} \leq|X| \leq 2(n-1) !
$$

We now proceed to the key step of the proof.

Theorem 22. If $\rho$ is a partition of $n$, let $X_{\rho}$ denote the conjugacy class of permutations with cycle-type $\rho$. Assume that $n>3 k+1$, and let $q_{k}$ denote the number of partitions $\geq\left(n-k, 1^{k}\right)$. Let $\phi_{1}, \ldots, \phi_{q_{k}-1}$ be the partitions of $n$ which are $>\left(n-k, 1^{k}\right)$ (in decreasing lexicographic order). Let $\mu_{1}, \ldots, \mu_{q_{k}-1}$ be such that for all $j$, either $\mu_{j}=\phi_{j}$ or else $\mu_{j}=\operatorname{Split}\left(\phi_{j}\right)$. Let $G_{j}=\operatorname{Cay}\left(S_{n}, X_{\mu_{j}}\right)$, and let $\left(\lambda_{i}^{(j)}\right)_{i}$ be the eigenvalues of $G_{j}$, in decreasing lexicographic order of their representations (so that $\lambda_{1}^{(j)}=\left|X_{\mu_{j}}\right|$ ).

Then there exist $d_{1}, \ldots, d_{q_{k}-1} \in \mathbb{R}$ such that

$$
\sum_{j} d_{j} \lambda_{i}^{(j)}= \begin{cases}1, & \text { for } i=1 \\ \omega, & \text { for } 1<i \leq q_{k} .\end{cases}
$$

Moreover, there exists $B_{k}>0$ depending only on $k$ such that

$$
\max _{j}\left|d_{j}\right| \leq \frac{B_{k}}{(n-1) !} .
$$

Theorem 22 will enable us to get the correct eigenvalues on the fat representations, but we will also need the following, which ensures that the eigenvalues for the medium representations are smaller in absolute value:

Theorem 23. Under the assumptions and notation of Theorem 22, let $\rho$ be a medium partition, and let $\lambda_{\rho}=\sum_{j=1}^{q_{k}-1} d_{j} \lambda_{\rho}^{(j)}$. Then

$$
\left|\lambda_{\rho}\right| \leq c_{k}|\omega| / \sqrt{n}=o(|\omega|),
$$

where $c_{k}>0$ depends only on $k$.

We defer the proof of this until later and turn first to the proof of Theorem 22.

Proof of Theorem 22. There are three steps in the proof. Step 1 is showing the existence of coefficients $d_{j}$ such that equation (25) holds for $i<q_{k}$. Step 2 is showing that with these coefficients, the equation also holds for $i=q_{k}$. Step 3 is obtaining the bound on $\max _{j}\left|d_{j}\right|$.

Step $1\left(i<q_{k}\right)$ : Consider the system of linear equations $M d=b$, where $d=\left(d_{1}, \ldots, d_{q_{k}-1}\right), b=(1, \omega, \ldots, \omega)$, and $M_{j i}=\lambda_{i}^{(j)}$. By Theorem 10,

$$
\lambda_{i}^{(j)}=\frac{1}{\operatorname{dim}\left[\phi_{i}\right]} \sum_{\tau \in X_{j}} \chi_{\phi_{i}}(\tau)=\frac{\left|X_{j}\right|}{\operatorname{dim}\left[\phi_{i}\right]} \chi_{\phi_{i}}\left(\tau_{j}\right),
$$

where $\tau_{j}$ is a representative for the conjugacy class $X_{j}$ (recall that characters are class functions). Equivalently,

$$
M=N_{1} \breve{C}^{t} N_{2},
$$


where $\breve{C}$ denotes the minor of the character table of $S_{n}$ with rows $\phi_{1}, \ldots, \phi_{q_{k}-1}$ and columns $\mu_{1}, \ldots, \mu_{q_{k}-1}$, and $N_{1}$ and $N_{2}$ are the diagonal row- and columnnormalization matrices respectively; explicitly,

$$
\left(N_{1}\right)_{i, j}=\frac{\delta_{i, j}}{\operatorname{dim}\left[\phi_{i}\right]}, \quad\left(N_{2}\right)_{i, j}=\delta_{i, j}\left|X_{j}\right|
$$

Recall from Theorem 21 that $\breve{C}$ is always equal to the top-left minor $\tilde{C}$, and that $\tilde{C}$ (and therefore $\tilde{C}^{t}$ ) is invertible. Therefore, there is a (unique) solution for $d$, so we can find appropriate values for $d_{1}, \ldots, d_{q_{k}-1}$.

Step $2\left(i=q_{k}\right)$ : Write

$$
\lambda=\sum_{j} d_{j} \lambda_{q_{k}}^{(j)} .
$$

We must show that $\lambda=\omega$. This will follow from analyzing the proof of the generalized Hoffman bound, Theorem 12 , when $G=\Gamma_{k}$ and the independent set $I$ is a $k$-coset.

Let $T$ be a $k$-coset of $S_{n}$, and let $f=1_{T}$ be its characteristic function. Choose the orthonormal basis of eigenvectors $\left\{v_{i}\right\}$ in Theorem 12 to be the normalization of the orthogonal basis formed by the entries of (matrix equivalents of) the irreducible representations $[\rho]$ (see section $[2.3)$. Observe that

$$
\alpha=\mathbb{E} f=\frac{(n-k) !}{n !} .
$$

By the argument in the proof of Theorem [7, we see that

$$
\widehat{f}(\rho) \neq 0 \Rightarrow \rho \geq\left(n-k, 1^{k}\right)
$$

and moreover, $\widehat{f}\left(\left(n-k, 1^{k}\right)\right) \neq 0$.

Let $W(\rho)=\operatorname{Tr}\left[\hat{f}(\rho)(\hat{f}(\rho))^{t}\right]$. This is simply the $L^{2}$-weight of the coefficients of eigenvectors $v_{i}$ which correspond to entries of (the matrix equivalent of) [ $\rho$ ]; i.e., if we write as usual $f=\sum a_{i} v_{i}$, then $W(\rho)=\sum a_{i}^{2}$, where the sum is over all $i$ such that $v_{i}$ is (the normalization of) one of the $(\operatorname{dim} \rho)^{2}$ eigenvectors which are entries of $[\rho]$. Hence,

$$
W(\rho) \neq 0 \Rightarrow \rho \geq\left(n-k, 1^{k}\right),
$$

and moreover, $W\left(\left(n-k, 1^{k}\right)\right) \neq 0$.

Using the coefficients $d_{1}, \ldots, d_{q_{k}-1}$ in Theorem 12, we have $\lambda_{1}=1$ and $\lambda_{t}=\omega$ for $1<t<q_{k}$. Since $T$ is an independent set in the graph $G=\Gamma_{k}$, we have

$$
\begin{aligned}
0 & =\sum_{\rho \geq\left(n-k, 1^{k}\right)} \lambda_{\rho} W(\rho) \\
& =\alpha^{2}+\omega\left(\alpha-\alpha^{2}-W\left(\left(n-k, 1^{k}\right)\right)\right)+\lambda W\left(\left(n-k, 1^{k}\right)\right) .
\end{aligned}
$$

By definition of $\omega$, we have $\omega\left(\alpha-\alpha^{2}\right)=-\alpha^{2}$; combining this with the above yields $\lambda=\omega$, as required.

Step 3 (bounding the coefficients $d_{j}$ ): Recall that $M=N_{1} \tilde{C}^{t} N_{2}$, and by Theorem 21 $\tilde{C}$ is invertible and independent of $n$. Hence, the entries of $\left(\tilde{C}^{t}\right)^{-1}$ are uniformly bounded by some function of $k$ alone. Since $M d=b$, we have

$$
d=M^{-1} b=\left(N_{2}\right)^{-1}\left(\tilde{C}^{t}\right)^{-1}\left(N_{1}^{-1} b\right) .
$$

We now proceed to bound uniformly the entries of the vector $N_{1}^{-1} b$. We have

$$
\left(N_{1}^{-1}\right)_{i j}=\delta_{i j} \operatorname{dim}\left[\phi_{i}\right],
$$


and therefore

$$
\left(N_{1}^{-1} b\right)_{i}=\operatorname{dim}\left[\phi_{i}\right] b_{i}
$$

For $i=1$, we have

$$
\left(N_{1}^{-1} b\right)_{1}=\operatorname{dim}\left[\phi_{1}\right] b_{1}=1 \cdot 1=1
$$

For $i>1$, we have

$$
\operatorname{dim}\left[\phi_{i}\right]+1 \leq \operatorname{dim}\left(M^{\left(n-k, 1^{k}\right)}\right)=n(n-1) \ldots(n-k+1),
$$

since both $\phi_{i}$ and the trivial representation are constituents of $M^{\left(n-k, 1^{k}\right)}$. Hence,

$$
\left|\left(N_{1}^{-1} b\right)_{i}\right|=\left|\operatorname{dim}\left[\phi_{i}\right]\right||\omega| \leq 1 .
$$

We now bound uniformly the entries of the matrix $\left(N_{2}\right)^{-1}$. Using Lemma 5, we have

$$
\left(N_{2}^{-1}\right)_{i j}=\frac{\delta_{i j}}{\left|X_{j}\right|} \leq \frac{(n-2 k-1)(2 k-1)^{2 k-1}}{n !} \leq \frac{b_{k}}{(n-1) !},
$$

where $b_{k}>0$ depends only on $k$.

Combining these bounds with (29), we see that there exists $B_{k}>0$ depending only on $k$ such that

$$
\left|d_{j}\right| \leq \frac{B_{k}}{(n-1) !} \forall j<q_{k}
$$

completing the proof.

Next, we need two more lemmas to assist in the proof of Theorem 23 .

Lemma 6. Let $G$ be a finite group, let $X \subset G$ be inverse-closed and conjugationinvariant, and let $\operatorname{Cay}(G, X)$ be the Cayley graph on $G$ with generating set $X$. Let $\rho$ be an irreducible representation of $G$ with dimension $d$, and let $\lambda_{\rho}$ be the corresponding eigenvalue of $\operatorname{Cay}(G, X)$, as in Theorem 10. Then

$$
\left|\lambda_{\rho}\right| \leq \frac{\sqrt{|G||X|}}{d}
$$

Proof. Since the irreducible characters of $G$ are orthonormal, we have

$$
\left\langle\chi_{\rho}, \chi_{\rho}\right\rangle=\frac{1}{|G|} \sum_{g \in G}\left|\chi_{\rho}(g)\right|^{2}=1 \text {. }
$$

By the Cauchy-Schwarz inequality,

$$
\left|\left\langle\chi_{\rho}, 1_{X}\right\rangle\right| \leq \sqrt{\left\langle\chi_{\rho}, \chi_{\rho}\right\rangle\left\langle 1_{X}, 1_{X}\right\rangle}=\sqrt{|X| /|G|}
$$

Substituting into Equation [7, we obtain

$$
\left|\lambda_{\rho}\right|=\frac{|G|\left|\left\langle\chi_{\rho}, 1_{X}\right\rangle\right|}{d} \leq \frac{\sqrt{|G||X|}}{d}
$$

as required.

Combining this with Lemma 5 yields:

Lemma 7. Let $X$ be a conjugacy class of $S_{n}$, with cycle-type having a cycle of length at least $n-2 k-1$. Let $\operatorname{Cay}\left(S_{n}, X\right)$ be the Cayley graph on $S_{n}$ generated by $X$. For any $C_{k}>0$, there exists $D_{k}>0$, depending only on $k$, such that if $\rho$ is 
an irreducible representation of dimension at least $C_{k} n^{k+1}$, then the corresponding eigenvalue $\lambda_{\rho}$ of $\operatorname{Cay}\left(S_{n}, X\right)$ satisfies

$$
\left|\lambda_{\rho}\right|<D_{k} \frac{(n-1) !\left|\omega_{n, k}\right|}{\sqrt{n}} .
$$

Proof. Note that $\left|\omega_{n, k}\right|=\Theta\left(1 / n^{k}\right)$. By choosing $D_{k}$ large enough, we may assume that $n>4 k+2$, so $n-2 k-1>n / 2$, and therefore the hypotheses of Lemma 5 hold. Assume that $\operatorname{dim} \rho \geq C_{k} n^{k+1}$. Then

$$
\begin{aligned}
\left|\lambda_{\rho}\right| & \leq \frac{\sqrt{|G||X|}}{C_{k} n^{k+1}} \\
& \leq \frac{\sqrt{2 n !(n-1) !}}{C_{k} n^{k+1}} \\
& =\frac{\sqrt{2}(n-1) !}{C_{k} n^{k+1 / 2}} \\
& \leq D_{k} \frac{(n-1) !\left|\omega_{n, k}\right|}{\sqrt{n}}
\end{aligned}
$$

as required.

We can now prove Theorem 23

Proof of Theorem 23. Assume the hypotheses of Theorem 23. By Lemma 2, we have $\operatorname{dim}[\rho] \geq E_{k} n^{k+1}$. Hence, we see that

$$
\begin{aligned}
\left|\lambda_{\rho}\right| & =\left|\sum_{j=1}^{q_{k}-1} d_{j} \lambda_{\rho}^{(j)}\right| \\
& \leq\left(q_{k}-1\right) \max _{j}\left|d_{j}\right| \max _{j}\left|\lambda_{\rho}^{(j)}\right| \\
& \leq\left(q_{k}-1\right) \frac{B_{k}}{(n-1) !} D_{k} \frac{(n-1) !\left|\omega_{n, k}\right|}{\sqrt{n}} \\
& =c_{k}|\omega| / \sqrt{n},
\end{aligned}
$$

as required.

4.3. The linear combination of Cayley graphs. Finally, we can now construct the linear combination of Cayley graphs we will use to prove our main theorem.

Theorem 24. There exists a linear combination $Y_{\text {even }}$ of Cayley graphs generated by conjugacy classes of even permutations within $F P F_{k}$, such that its eigenvalues are as described in the first line of Table 1, i.e.,

- $\lambda_{(n)}=1$,

- $\lambda_{\rho}=\omega$ for each fat $\rho \neq(n)$,

- $\lambda_{\left(1^{n}\right)}=1$,

- $\lambda_{\rho}=\omega$ for each tall $\rho \neq\left(1^{n}\right)$, and

- $\left|\lambda_{\rho}\right| \leq c_{k}|\omega| / \sqrt{n} \leq o(|\omega|)$ for each medium $\rho$, where $c_{k}>0$ depends only on $k$. 
Proof. Recall that for each partition $\phi_{j}>\left(n-k, 1^{k}\right)$, exactly one of $\phi_{j}$ and $\operatorname{Split}\left(\phi_{j}\right)$ is even. For each partition $\phi_{j}>\left(n-k, 1^{k}\right)$, define

$$
\mu_{j}=\left\{\begin{aligned}
\phi_{j}, & \text { if } X_{\phi_{j}} \text { is an even conjugacy class; } \\
\operatorname{Split}\left(\phi_{j}\right), & \text { if } X_{\phi_{j}} \text { is an odd conjugacy class. }
\end{aligned}\right.
$$

Then each $X_{\mu_{j}}$ consists of even permutations. Take

$$
Y_{\text {even }}=\sum_{j=1}^{q_{k}-1} d_{j} \operatorname{Cay}\left(S_{n}, X_{\mu_{j}}\right),
$$

where the $d_{j}$ 's are as defined in Theorem 22. Then we have $\lambda_{(n)}=1$, and for each fat $\rho \neq(n)$, we have $\lambda_{\rho}=\omega$. By Theorem 17, for any partition $\rho$, we have $\chi_{\rho^{t}}=\chi_{\rho} \cdot$ sgn. So by equation (24), for any partition $\rho$, we have $\lambda_{\rho^{t}}=\lambda_{\rho}$, since our Cayley graphs are all generated by conjugacy classes of even permutations. Therefore, $\lambda_{\left(1^{n}\right)}=1$, and $\lambda_{\rho}=\omega$ for each tall $\rho \neq\left(1^{n}\right)$. By Theorem 23, $\left|\lambda_{\rho}\right| \leq c_{k}|\omega| / \sqrt{n} \leq o(|\omega|)$ for each medium $\rho$, completing the proof.

Theorem 25. There exists a linear combination $Y_{\text {odd }}$ of Cayley graphs generated by conjugacy classes of odd permutations within $F P F_{k}$, such that its eigenvalues satisfy

- $\lambda_{(n)}=1$,

- $\lambda_{\rho}=\omega$ for all fat $\rho \neq(n)$,

- $\lambda_{\left(1^{n}\right)}=-1$,

- $\lambda_{\rho}=-\omega$ for each tall $\rho \neq\left(1^{n}\right)$, and

- $\left|\lambda_{\rho}\right| \leq c_{k}|\omega| / \sqrt{n} \leq o(|\omega|)$ for each medium $\rho$, where $c_{k}>0$ depends only on $k$.

Proof. For each partition $\phi_{j}>\left(n-k, 1^{k}\right)$, define

$$
\mu_{j}=\left\{\begin{aligned}
\phi_{j}, & \text { if } X_{\phi_{j}} \text { is an odd conjugacy class; } \\
\operatorname{Split}\left(\phi_{j}\right), & \text { if } X_{\phi_{j}} \text { is an even conjugacy class. }
\end{aligned}\right.
$$

Then each $X_{\mu_{j}}$ consists of odd permutations. Take

$$
Y_{\text {odd }}=\sum_{j=1}^{q_{k}-1} d_{j} \operatorname{Cay}\left(S_{n}, X_{\mu_{j}}\right),
$$

where the $d_{j}$ 's are as defined in Theorem 22. Then we have $\lambda_{(n)}=1$, and for each fat $\rho \neq(n)$, we have $\lambda_{\rho}=\omega$. This time, our Cayley graphs are all generated by odd permutations, so we have $\lambda_{\rho^{t}}=-\lambda_{\rho}$ for every partition $\rho$. Hence, $\lambda_{\left(1^{n}\right)}=-1$, and $\lambda_{\rho}=-\omega$ for each tall $\rho \neq\left(1^{n}\right)$. Again, by Theorem 23, $\left|\lambda_{\rho}\right| \leq c_{k}|\omega| / \sqrt{n} \leq o(|\omega|)$ for each medium $\rho$, completing the proof.

Theorem 26. There exists a linear combination $Y$ of Cayley graphs generated by conjugacy classes within $F P F_{k}$, such that its eigenvalues satisfy:

- $\lambda_{(n)}=1$,

- $\lambda_{\rho}=\omega$ for each fat $\rho \neq(n)$,

- $\lambda_{\rho}=0$ for each tall $\rho$, and

- $\left|\lambda_{\rho}\right| \leq c_{k}|\omega| / \sqrt{n} \leq o(|\omega|)$ for each medium $\rho$, where $c_{k}>0$ depends only on $k$.

Proof. Set $Y=\frac{1}{2}\left(Y_{\text {even }}+Y_{\text {odd }}\right)$. 
Proof of Theorems 5 and 6. If $n$ is sufficiently large depending on $k$, then $c_{k}|\omega| / \sqrt{n}$ $<|\omega|$, so $\omega$ is both the minimum eigenvalue of $Y$ and the second largest in absolute value. By our choice of $\omega$, applying the generalized Hoffman Theorem 12 to $Y$, we see that if $I \subset S_{n}$ is $k$-intersecting, then $|I| \leq(n-k)$ !, and that if equality holds, then the characteristic function $1_{I} \in V_{k}$. Similarly, applying Theorem 13 to $Y$, we see that if $I, J \subset S_{n}$ are $k$-cross-intersecting, then $|I||J| \leq((n-k) !)^{2}$, and that if equality holds, then $1_{I}, 1_{J} \in V_{k}$.

\section{Boolean functions}

Recall that $V_{k}$ is the linear space of real-valued functions on $S_{n}$ whose Fourier transform is supported only on irreducible representations $\geq\left(n-k, 1^{k}\right)$. Theorem 7 states that $V_{k}$ is spanned by the $k$-cosets of $S_{n}$. We now wish to characterize the Boolean functions in $V_{k}$, proving (a strengthened version of) Theorem 8 ,

We will show that every nonnegative function in $V_{k}$ can be written as a linear combination of the characteristic functions of $k$-cosets with nonnegative coefficients, and every $0 / 1$ valued function in $V_{k}$ can be written with $0 / 1$ coefficients.

Let $\mathcal{A}_{k}$ be the set of ordered $k$-tuples of distinct numbers between 1 and $n$, and let $(n)_{k}=\left|\mathcal{A}_{k}\right|=\frac{n !}{(n-k) !}=n(n-1) \ldots(n-k+1)$. We will prove the following.

Theorem 27. Let $f \in V_{k}$ be nonnegative. Then there exist nonnegative coefficients $\left(b_{\alpha, \beta}\right)_{\alpha, \beta \in \mathcal{A}_{k}}$ such that $f=\sum b_{\alpha, \beta} 1_{T_{\alpha \mapsto \beta}}$. Furthermore, if $f$ is Boolean, then $f$ is the characteristic function of a disjoint union of $k$-cosets.

For didactic reasons, we begin by dealing with the case $k=1$.

Theorem 28. If $f \in V_{1}$ is nonnegative, then there exist $b_{i, j} \geq 0$ such that $f=$ $\sum_{i, j} b_{i, j} 1_{T_{i \mapsto j}}$.

Proof. We say that a real $n \times n$ matrix $A=\left(a_{i, j}\right)_{i, j \in[n]}$ represents a function $f \in V_{1}$ if

$$
f=\sum_{i, j} a_{i, j} 1_{T_{i \mapsto j}}
$$

or equivalently,

$$
f(\sigma)=\sum_{i=1}^{n} a_{i, \sigma(i)} \quad \forall \sigma \in S_{n} .
$$

Let $A$ be a matrix representing $f$. Our task is to find a nonnegative matrix $B$ which also represents $f$. Let $x_{1}, \ldots, x_{n}$ and $y_{1}, \ldots, y_{n}$ be real numbers such that

$$
\sum_{i=1}^{n} x_{i}+\sum_{j=1}^{n} y_{j}=0
$$

Let $B=\left(b_{i, j}\right)_{i, j \in[n]}$ be the matrix produced from $A$ by adding $x_{i}$ to row $i$ for each $i$, and then adding $y_{j}$ to column $j$ for each $j$, i.e.

$$
b_{i, j}=a_{i, j}+x_{i}+y_{j} \quad(i, j \in[n]) .
$$

Note that $B$ also represents $f$. We wish to show that there exists a choice of $x_{i}$ 's and $y_{j}$ 's such that the matrix $B$ has all its entries nonnegative; i.e., we wish to solve the system of inequalities

$$
x_{i}+y_{j} \geq-a_{i, j}(1 \leq i, j \leq n) \quad \text { subject to } \quad \sum_{i} x_{i}+\sum_{j} y_{j}=0 .
$$


By the strong duality theorem of linear programming (see 23]), this is unsolvable if and only if there exist $c_{i, j} \geq 0$ such that

$$
\sum_{j} c_{i, j}=1(1 \leq i \leq n), \quad \sum_{i} c_{i, j}=1(1 \leq j \leq n), \quad \sum_{i, j} c_{i, j} a_{i, j}<0 .
$$

Suppose for a contradiction that this holds. The matrix $C=\left(c_{i, j}\right)_{i, j \in[n]}$ is bistochastic, and therefore by Birkhoff's theorem (see [4]), it can be written as a convex combination of permutation matrices,

$$
C=\sum_{t=1}^{r} s_{t} P_{\sigma_{t}}
$$

where $s_{t} \geq 0(1 \leq t \leq r), \sum_{t=1}^{r} s_{t}=1, \sigma_{1}, \ldots, \sigma_{t} \in S_{n}$, and $P_{\sigma}$ denotes the permutation matrix of $\sigma$, i.e. $\left(P_{\sigma}\right)_{i, j}=1_{\{\sigma(i)=j\}}$. But then $\sum_{i, j} c_{i, j} a_{i, j}$ is a convex combination of values of $f$ :

$$
\sum_{i, j} c_{i, j} a_{i, j}=\sum_{t=1}^{r} s_{t} \sum_{i, j}\left(P_{\sigma_{t}}\right)_{i, j} a_{i, j}=\sum_{t=1}^{r} s_{t} \sum_{i=1}^{n} a_{i, \sigma_{t}(i)}=\sum_{t=1}^{r} s_{t} f\left(\sigma_{t}\right)
$$

and is therefore nonnegative, a contradiction. Hence, the system (30) is solvable, proving the theorem.

The following corollary is immediate.

Corollary 2. If $f \in V_{1}$ is Boolean, then it is the characteristic function of a disjoint union of 1-cosets.

Proof. The proof is by induction on the number of nonzero values of $f$. Let $f \in V_{1}$ be a Boolean function and suppose the statement is true for all Boolean functions with fewer nonzero values. By Theorem 28 there exist $b_{i, j} \geq 0$ such that

$$
f=\sum_{i, j} b_{i, j} 1_{T_{i \mapsto j}} .
$$

Choose $i, j$ such that $b_{i, j}>0$. Then $f>0$ on $T_{i \mapsto j}$, so $f=1$ on $T_{i, j}$. Hence, $f-1_{T_{i \mapsto j}}$ is also Boolean, with fewer nonzero values than $f$, and so by the induction hypothesis, it is the characteristic function of a disjoint union of 1-cosets. Hence, the same is true of $f$, as required.

We now extend this proof to the case $k>1$, proving Theorem 27. However, some preliminaries are necessary.

Let $A$ be an $(n)_{k} \times(n)_{k}$ matrix with rows and columns indexed by $\mathcal{A}_{k}$. Choose an ordering of the $k$ coordinates of the $k$-tuples, or equivalently a permutation $\pi \in S_{k}$, and consider the natural lexicographic ordering on $\mathcal{A}_{k}$ induced by this ordering, i.e. $\alpha<\beta$ iff $\alpha_{\pi(m)}<\beta_{\pi(m)}$, where $m=\min \left\{l: \alpha_{\pi(l)} \neq \beta_{\pi(l)}\right\}$. This lexicographic ordering on $\mathcal{A}_{k}$ recursively partitions $A$ into blocks: first it partitions $A$ into $n^{2}$ $(n-1)_{k-1} \times(n-1)_{k-1}$ blocks $B_{i, j}$ according to the $\pi(1)$-coordinate of each $k$-tuple; then it partitions each block $B_{i, j}$ into $(n-1)^{2}(n-2)_{k-2} \times(n-2)_{k-2}$ sub-blocks according to the $\pi(2)$-coordinate of each $k$-tuple, and so on.

The following two recursive definitions concerning such matrices will be crucial.

Definition 13. We define a $k$-line in an $(n)_{k} \times(n)_{k}$ matrix $A$ as follows. If $k=1$, i.e. if $A$ is an $n \times n$ matrix, a 1-line in $A$ is just a row or column of $A$. If $k>1$, and $A$ is an $(n)_{k} \times(n)_{k}$ matrix, a $k$-line in $A$ is given by choosing an ordering $\pi$ of 
the $k$-coordinates, partitioning $A$ into $n^{2}$ blocks according to the $\pi(1)$-coordinate of each $k$-tuple as above, choosing a row or column of $(n-1)_{k-1} \times(n-1)_{k-1}$ blocks $B_{i, j}$, and then taking a union of $(k-1)$-lines, one from each block.

Definition 14. We say that an $(n)_{k} \times(n)_{k}$ matrix $A$ is $k$-bistochastic if the following holds. If $k=1$, an $n \times n$ matrix $A$ is 1-bistochastic if it is bistochastic in the usual sense, i.e. all its entries are nonnegative, and all its row and column sums are 1 . If $k>1$, an $(n)_{k} \times(n)_{k}$ matrix $A$ is $k$-bistochastic if, for any partition into $n^{2}$ blocks $B_{i, j}$ of size $(n-1)_{k-1} \times(n-1)_{k-1}$ according to a lexicographic order on $\mathcal{A}_{k}$ induced by any of the $k$ ! orderings of the coordinates, there exists a bistochastic $n \times n$ matrix $R=\left(r_{i, j}\right)$ and $n^{2}(k-1)$-bistochastic matrices $M_{i, j}$ of order $(n-1)$, such that $B_{i, j}=r_{i, j} M_{i, j}$.

The following two self-evident observations indicate the relevance of these two notions.

Observation 1. An $(n)_{k} \times(n)_{k}$ matrix with nonnegative entries is $k$-bistochastic if and only if the sum of the entries of every $k$-line in it (coming from any of the $k$ ! recursive partitions into blocks) is 1 .

Observation 2. Given a $k$-line $\ell$, the corresponding $k$-cosets $\left\{T_{\alpha \mapsto \beta}:(\alpha, \beta) \in \ell\right\}$ partition $S_{n}$.

Any permutation $\sigma \in S_{n}$ induces a permutation of $\mathcal{A}_{k}$; we write $P_{\sigma}^{(k)}$ for the corresponding $(n)_{k} \times(n)_{k}$ permutation matrix, i.e.

$$
\left(P_{\sigma}^{(k)}\right)_{\alpha, \beta}=1_{\{\sigma(\alpha)=\beta\}} .
$$

These $n$ ! permutations are only a small fraction of all $\left((n)_{k}\right)$ ! permutations of $\mathcal{A}_{k}$. It is not hard to show that any $P_{\sigma}^{(k)}$ is $k$-bistochastic. It is a bit harder to show that any other permutation matrix is not $k$-bistochastic (for this it is important to note that we demand taking into account lexicographic orderings induced by all possible orderings of the coordinates).

The authors thank Siavosh Benabbas for suggesting the correct formulation of the following theorem and for help in proving it.

Theorem 29 (Generalized Birkhoff theorem [Benabbas, Friedgut, Pilpel]). An $(n)_{k}$ $\times(n)_{k}$ matrix $M$ is $k$-bistochastic if and only if it is a convex combination of $(n)_{k} \times(n)_{k}$ matrices of permutations of $\mathcal{A}_{k}$ which are induced by permutations of $[n]$.

Proof. By induction on $k$.

For $k=1$, this is Birkhoff's theorem.

For the induction step, let $M=r_{i, j} \cdot M_{i, j}$ be a block decomposition of $M$ as in the definition of $k$-bistochasticity, according to the natural ordering of the coordinates, i.e. $\pi=\mathrm{id}$. By Birkhoff's theorem, $R=\left(r_{i, j}\right)$ is a convex combination of permutation matrices, and therefore it is either a permutation matrix, or else it can be written as a convex combination $R=s P+(1-s) T$, with $P$ a permutation matrix, and $T$ a bistochastic matrix with more zero entries than $R$. Treating $P$ separately, and proceeding in this manner by induction, we may assume that $R$ is a permutation matrix, and without loss of generality, we may assume that $R=I$, the identity matrix. So in $M$, every nonzero entry is indexed by a pair of $k$-tuples of the form $((i, *, *, \ldots, *),(i, *, *, \ldots, *))$ for some $i$. 
Now, we reorder the rows and columns of $M$ with a lexicographic order on $\mathcal{A}_{k}$ determined by the transposition $\pi=\left(\begin{array}{l}1 \\ 2\end{array}\right)$. Consider now an off-diagonal block in this ordering of the form $((*, i, *, *, \ldots, *),(*, j, *, *, \ldots, *))$ with $i \neq j$. It breaks into $(n-1)^{2}$ sub-blocks of size $(n-2)_{k-2} \times(n-2)_{k-2}$, but only $n-2$ of them have nonzero entries, namely the sub-blocks of the form $((l, i, *, *, \ldots, *),(l, j, *, *, \ldots, *))$ for some $l \neq i, j$. Hence, the block has a row of zero sub-blocks, namely the subblocks

$$
((j, i, *, *, \ldots, *),(m, j, *, *, \ldots, *))
$$

for $m \neq j$, and a column of zero sub-blocks, namely the sub-blocks

$$
((m, i, *, *, \ldots, *),(i, j, *, *, \ldots, *))
$$

for $m \neq i$. Since the block is $(k-1)$-bistochastic, it must be the zero matrix.

As for the diagonal blocks of $M$ in the new ordering of $\mathcal{A}_{k}$, blocks of the form $((*, i, *, *, \ldots, *),(*, i, *, *, \ldots, *))$, they can only have nonzero sub-blocks on the diagonal. Since they are $(k-1)$-bistochastic, by the induction hypothesis they are each equal to a convex linear combination of $P_{\sigma}^{(k-1)}$ 's where each $\sigma$ is a permutation of $[n] \backslash\{i\}$. But if any of these $\sigma$ 's has $\sigma(l) \neq l$ for some $l$, then the off-diagonal sub-block

$$
((l, i, *, *, \ldots, *),(\sigma(l), i, *, *, \ldots, *))
$$

is nonzero, a contradiction. Hence, each permutation $\sigma$ must be the identity, and therefore all the diagonal blocks are copies of the $(n-1)_{k-1} \times(n-1)_{k-1}$ identity matrix. Hence, $M$ is the identity matrix, and we are done.

We now can prove Theorem 27

Proof of Theorem 27. We follow the lines of the proof of Theorem 28. Let $f \in V_{k}$ be nonnegative, and let $M=\left(M_{\alpha, \beta}\right)_{\alpha, \beta \in \mathcal{A}_{k}}$ be an $(n)_{k} \times(n)_{k}$ matrix (with rows and columns indexed by $\mathcal{A}_{k}$ ) which represents $f$, meaning that

$$
f=\sum_{\alpha, \beta \in \mathcal{A}_{k}} M_{\alpha, \beta} 1_{T_{\alpha \mapsto \beta}},
$$

i.e.

$$
f(\sigma)=\sum_{\alpha \in \mathcal{A}_{k}} M_{\alpha, \sigma(\alpha)} \quad \forall \sigma \in S_{n} .
$$

Let $\mathcal{L}_{k}$ be the set of all $k$-lines. Recall from Observation 2 that every $k$-line corresponds to a partition of $S_{n}$ into $k$-cosets. Therefore, adding a constant $x \in \mathbb{R}$ to a single $k$-line in $M$ corresponds to increasing $f$ by $x$. We therefore associate with every line $\ell \in \mathcal{L}_{k}$ a variable $x_{\ell}$. We wish to solve the following system of linear inequalities:

$$
\sum_{\ell:(\alpha, \beta) \in \ell} x_{\ell} \geq-M_{\alpha, \beta}\left(\ell \in \mathcal{L}_{k}\right) \quad \text { subject to } \quad \sum_{\ell \in \mathcal{L}_{k}} x_{\ell}=0 .
$$

Again, by the strong duality theorem of linear programming, this is possible unless there exists an $(n)_{k} \times(n)_{k}$ matrix $C=\left(c_{\alpha, \beta}\right)_{\alpha, \beta \in \mathcal{A}_{k}}$ with nonnegative entries, such that

$$
\sum_{(\alpha, \beta) \in \ell} c_{\alpha, \beta}=1 \quad \forall \ell \in \mathcal{L}_{k}
$$

and

$$
\sum_{\alpha, \beta \in \mathcal{A}_{k}} c_{\alpha, \beta} M_{\alpha, \beta}<0 .
$$


By Observation 1. $C$ is $k$-bistochastic. By the Generalized Birkhoff Theorem, $C$ is in the convex hull of the permutation matrices induced by permutations of $[n]$. This means, as before, that

$$
\sum_{\alpha, \beta \in \mathcal{A}_{k}} c_{\alpha, \beta} M_{\alpha, \beta}
$$

is a convex linear combination of values of $f$, and is therefore nonnegative, a contradiction. Therefore, the system of linear inequalities above is solvable, proving the first part of the theorem. The second part follows as in the $k=1$ case, by induction on the number of nonzero values of $f$.

This completes the proof of Theorems 3 and 4 .

\section{OPEN PROBLEMS}

In their landmark paper [1, Ahlswede and Khachatrian characterized the largest $k$-intersecting subsets of $[n]^{(r)}$ for every value of $k, r$ and $n$. In our opinion, the most natural open problem in the area is to characterize the largest $k$-intersecting subsets of $S_{n}$ for every value of $n$ and $k$. We make the following.

Conjecture 2. A $k$-intersecting family in $S_{n}$ with maximum size must be a 'double translate' of one of the families

$$
M_{i}=\left\{\sigma \in S_{n}: \sigma \text { has } \geq k+i \text { fixed points in }[k+2 i]\right\}(0 \leq i \leq(n-k) / 2),
$$

i.e. of the form $\sigma M_{i} \tau$, where $\sigma, \tau \in S_{n}$.

This would imply that the maximum size is $(n-k)$ ! for $n>2 k$. It is natural to ask how large $n$ must be for our method to give this bound, i.e. when there exists a weighted graph $Y$ which is a real linear combination of Cayley graphs on $S_{n}$ generated by conjugacy classes in $\mathrm{FPF}_{k}$, such that $Y$ has maximum eigenvalue 1 and minimum eigenvalue

$$
\omega_{n, k}=-\frac{1}{n(n-1) \ldots(n-k+1)-1} .
$$

It turns out that this need not be the case when $n=2 k+2$; indeed, it fails for $k=2$ and $n=6$. We believe that new techniques will be required to prove Conjecture 2 ,

\section{ACKNOWLEDGEMENTS}

The authors would like to thank Noga Alon, Siavosh Benabbas, Imre Leader, Timothy Gowers, Yuval Roichman, and John Stembridge for various useful remarks.

\section{REFERENCES}

[1] Rudolf Ahlswede and Levon H. Khachatrian. The complete intersection theorem for systems of finite sets. European Journal of Combinatorics, 18(2):125-136, 1997. MR1429238 (97m:05251)

[2] Noga Alon, Haim Kaplan, Michael Krivelevich, Dahlia Malkhi, and Julien Stern. Scalable secure storage when half the system is faulty. In 27th International Colloquium on Automata, Languages and Programming, pages 576-587, 2000. MR.1795918(2001h:68033) 
[3] László Babai. Spectra of Cayley graphs. Journal of Combinatorial Theory, Series B, 27:180189, 1979. MR0546860 (81f:05090)

[4] G. Birkhoff. Three observations on linear algebra. Univ. Nac. Tucumán. Revista A., 5:147151, 1946. MR0020547(8:561a)

[5] Jean Bourgain. On the distribution of the Fourier spectrum of Boolean functions. Israel Journal of Mathematics, 131:269-276, 2002. MR1942312 (2003j:43009)

[6] Peter J. Cameron and C. Y. Ku. Intersecting families of permutations. European Journal of Combinatorics, 24:881-890, 2003. MR2009400 (2004g:20003)

[7] Persi Diaconis and Mehrdad Shahshahani. Generating a random permutation with random transpositions. Probability Theory and Related Fields, 57(2):159-179, 1981. MR626813 (82h:60024)

[8] David Ellis. Stability for $t$-intersecting families of permutations. To appear in Journal of Combinatorial Theory, Series A.

[9] J. S. Frame, G. B. Robinson, and R. M. Thrall. The Hook graphs of $S_{n}$. Canadian Journal of Mathematics, 6:316-325, 1954. MR0062127(15:931g)

[10] Peter Frankl and Mikhail Deza. On the maximum number of permutations with given maximal or minimal distance. Journal of Combinatorial Theory, Series A, 22(3):352-360, 1977. MR 0439648 (55:12534)

[11] Ehud Friedgut. Boolean functions with low average sensitivity depend on few coordinates. Combinatorica, 18(1):27-36, 1998. MR1645642 (99g:28021)

[12] Ehud Friedgut, Gil Kalai, and Assaf Naor. Boolean functions whose Fourier transform is concentrated on the first two levels and neutral social choice. Advances in Applied Mathematics, 29:427-437, 2002. MR 1942632 (2003j:26011)

[13] Ehud Friedgut and Haran Pilpel. Intersecting families of permutations: An algebraic approach. Talk given at the Oberwolfach Conference in Combinatorics, January 2008.

[14] Chris Godsil and Karen Meagher. A new proof of the Erdős-Ko-Rado theorem for intersecting families of permutations. European Journal of Combinatorics, 30(2):404-414, 2009. MR2489272(2010b:05008)

[15] G.H. Hardy, J.E. Littlewood, and G. Pólya. An Introduction to Inequalities. Cambridge University Press, 1988. MR944909 (89d:26016)

[16] A.J.W. Hilton and E.C. Milner. Some intersection theorems for systems of finite sets. Quarterly Journal of Mathematics, Oxford, 18:369-384, 1967. MR0219428 (36:2510)

[17] A. J. Hoffman. On eigenvalues and colorings of graphs. Graph Theory and its Applications, 1969. MR0284373 (44:1601)

[18] I. Martin Isaacs. Character Theory of Finite Groups. Academic Press, 1976. MR 0460423 $(57: 417)$

[19] C. Jordan. Traité des Substitutions et des Equations Algébriques. Gauthier-Villars, Paris, 1870 .

[20] Jeff Kahn, Gil Kalai, and Nathan Linial. The influence of variables on Boolean functions (extended abstract). In FOCS, pages 68-80. IEEE, 1988.

[21] Benoit Larose and Claudia Malvenuto. Stable sets of maximal size in Kneser-type graphs. European Journal of Combinatorics, 25(5):657-673, 2004. MR2061391 (2005c:05154)

[22] Imre Leader. Open Problems Session, British Combinatorial Conference, 2005.

[23] Jiří Matoušek and Bernd Gärtner. Understanding and Using Linear Programming. SpringerVerlag, Berlin, 2007.

[24] S. P. Mishchenko. Lower bounds on the dimensions of irreducible representations of symmetric groups and of the exponents of the exponential of varieties of Lie algebras. Matematicheski Sbornik, 187:83-94, 1996. MR1380205 (97d:17003)

[25] Paul Renteln. On the spectrum of the derangement graph. Electronic Journal of Combinatorics, 14, 2007, 17 pp. \#R82. MR2365981 (2008j:05217)

[26] Yuval Roichman. Upper bound on the characters of the symmetric groups. Inventiones Mathematicae, 125:451-485, 1996. MR 1400314 (97e:20014)

[27] Bruce E. Sagan. The Symmetric Group: Representations, Combinatorial Algorithms, and Symmetric Functions. Springer-Verlag, New York, 1991. [2nd revised printing, 2001]. MR:1824028 (2001m:05261)

[28] Jean-Pierre Serre. Linear Representations of Finite Groups. Springer-Verlag, Berlin, 1977. $\operatorname{MR} 0450380(56: 8675)$ 
Department of Pure Mathematics and Mathematical Statistics, University of CamBridge, Wilberforce Road, Cambridge, CB3 0WB England

Current address: St John's College, Cambridge, CB2 1TP, United Kingdom

Department of Mathematics, Hebrew University, 91904 Jerusalem, Israel, and Department of Mathematics, University of Toronto, 40 St. George Street, Toronto, OnTARIO M5S 2E4, CANADA

Department of Mathematics, Hebrew University, 91904 Jerusalem, Israel

Current address: Google, Inc., Levinstein Tower 26th Floor, 23 Manachem Begin St, 66183 Tel Aviv, Israel 